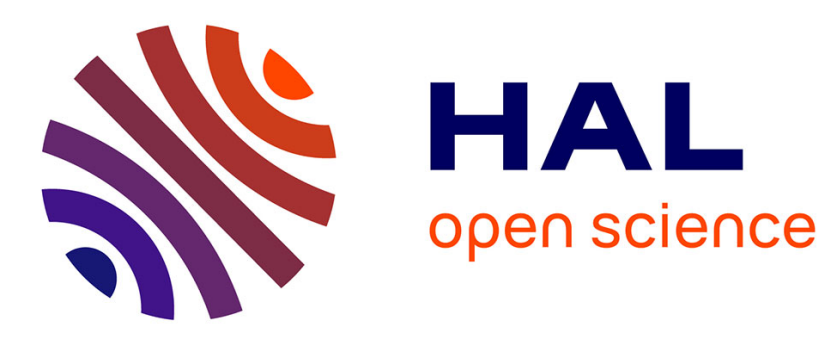

\title{
Modeling of polycrystals with gradient crystal plasticity - A comparison of strategies
}

\author{
Swantje Bargmann, Magnus Ekh, Kenneth Runesson, Bob Svendsen
}

\section{To cite this version:}

Swantje Bargmann, Magnus Ekh, Kenneth Runesson, Bob Svendsen. Modeling of polycrystals with gradient crystal plasticity - A comparison of strategies. Philosophical Magazine, 2010, 90 (10), pp.12631288. 10.1080/14786430903334332 . hal-00581028

\section{HAL Id: hal-00581028 \\ https://hal.science/hal-00581028}

Submitted on 30 Mar 2011

HAL is a multi-disciplinary open access archive for the deposit and dissemination of scientific research documents, whether they are published or not. The documents may come from teaching and research institutions in France or abroad, or from public or private research centers.
L'archive ouverte pluridisciplinaire HAL, est destinée au dépôt et à la diffusion de documents scientifiques de niveau recherche, publiés ou non, émanant des établissements d'enseignement et de recherche français ou étrangers, des laboratoires publics ou privés. 


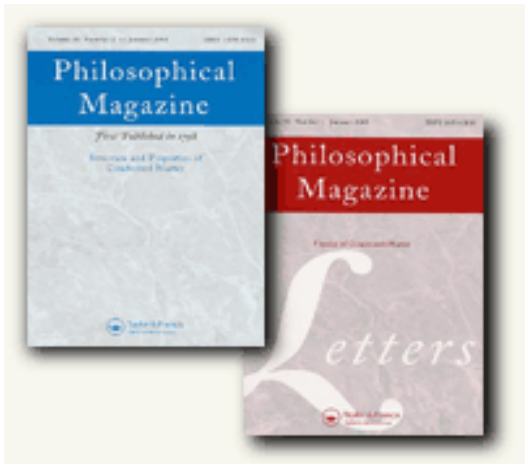

\section{Modeling of polycrystals with gradient crystal plasticity - A comparison of strategies}

\begin{tabular}{|r|l|}
\hline Journal: & Philosophical Magazine \& Philosophical Magazine Letters \\
\hline Manuscript ID: & TPHM-09-Mar-0092.R1 \\
\hline Journal Selection: & Philosophical Magazine \\
\hline Date Submitted by the & $29-J u l-2009$ \\
\hline Complete List of Authors: & $\begin{array}{l}\text { Bargmann, Swantje; University of Dortmund } \\
\text { Ekh, Magnus; Chalmers University of Technology } \\
\text { Runesson, Kenneth; Chalmers University of Technology } \\
\text { Svendsen, Bob; University of Dortmund }\end{array}$ \\
\hline Keywords: & computational mechanics, grain size, Hall effect \\
\hline Keywords (user supplied): & computational mechanics, grain size, Hall effect \\
\hline &
\end{tabular}

\section{S ScholaroNE}

Manuscript Central 


\title{
MANUSCRIPT
}

\section{Modeling of polycrystals with gradient crystal plasticity - A comparison of strategies}

\author{
Swantje Bargmann $^{\mathrm{a}}{ }^{*}$, Magnus Ekh $^{\mathrm{b}}$, Kenneth Runesson $^{\mathrm{b}}$, Bob Svendsen $^{\mathrm{a}}$ \\ ${ }^{a}$ Dortmund University of Technology, Chair of Mechanics, Department of Mechanical \\ Engineering, Leonhard-Euler-Str. 5, 44221 Dortmund, Germany; \\ ${ }^{\mathrm{b}}$ Chalmers University of Technology, Dept. of Applied Mechanics, Division of Material \\ and Computational Mechanics, 41296 Gothenburg, Sweden
}

(v4.4 released November 2008)

\begin{abstract}
This paper treats the computational modeling of size dependence in microstructure models of metals. Different gradient crystal plasticity strategies are analyzed and compared. For the numerical implementation, a dual-mixed finite element formulation which is suitable for parallelization is suggested. The paper ends with a representative numerical example for polycrystals.
\end{abstract}

Keywords: crystal plasticity; gradient hardening; grain modeling; dual mixed finite element; polycrystalline material; constitutive behavior

\section{Introduction}

It is well-known that the macroscopic behavior of a polycrystalline material is influenced by the size and morphology of the grains, the volume fraction of different phases, and the subgrain processes, for example. In order to include micro-effects in a macroscopic model, a complete scale separation is often assumed. With the help of a computational homogenization method the response of a Representative Volume Element (RVE), subjected to suitable boundary conditions that represent the macroscopic deformation (prolongation conditions), is coupled to the macroscopic level. However, conventional models of crystal plasticity, that are often used to model grain behavior, do not contain intrinsic material length-scales and are, therefore, not capable of capturing macroscopic size dependent effects. Thus, models including plastic strain gradients have been introduced in order to overcome this drawback of ordinary crystal plasticity theories. This contribution discusses different approaches to microstructure polycrystal models which include size dependence.

One size effect in metals is known as the Hall-Petch effect, i.e. the influence of the grain size on the macroscopic stress-strain characteristics. It is one of the important unresolved issues in computational material modeling. Hall [15] and Petch [20] independently studied different behaviors of steel, but eventually obtained the same result. While Hall [15] focused on factors influencing the mechanical properties of

${ }^{*}$ Corresponding author. Email: swantje.bargmann@uni-dortmund.de

ISSN: $1478-6435$ print/ISSN 1478-6443 online

(C) 2009 Taylor \& Francis

DOI: $10.1080 / 1478643$ YYxxxxxxxx

http://www.informaworld.com

http://mc.manuscriptcentral.com/pm-pml 
mild steels, Petch [20] studied brittle failure of steels. Nevertheless, Petch's results from 1953 are in excellent agreement with the ones of Hall which had been published two years earlier. The fact that the (current) flow stress $\sigma(\epsilon)$ depends on the mean grain size $d$ became known as the Hall-Petch effect:

$$
\sigma_{\mathrm{y}}(\epsilon)=\sigma_{\mathrm{y}, 0}(\epsilon)+\frac{k(\epsilon)}{\sqrt{d}}
$$

Both parameters, $\sigma_{\mathrm{y}, 0}$ and $k$, may depend on the strain level $\epsilon$ due to hardening of the material.

In the literature, different models have been developed in order to capture size dependence effects in crystals. These include thermodynamic approaches like $[7,13,14,17-19,22,24,25,27]$ as well as dislocation-field-theory-based approaches like [4] and [9]. Despite the large amount of models, there only exist few classifications or analyses of the common features and major differences. Examples which discuss similarities between Cosserat, strain gradient crystal plasticity and the statistical theory of dislocations in the case of single slip are the works of Forest and Sedláček [10,11]. A nonlocal plasticity theory is compared with a discrete dislocation model in [3] and [5]. Numerical results are presented for single slip in a two-dimensional composite material subject to plane strain simple shear. A boundary value problem for a single crystal undergoing small deformations is discussed for a phase field dislocation theory and Gurtin's theory (cf. [13]) in [16]. This contribution aims at comparing the three models presented in [7], [9] and a combination of [7] and [17].

The first model to be investigated in this contribution is the one introduced by Evers et al. [9] and continued by Bayley et al. [4]. As opposed to the two models mentioned below, it does not originate from a thermodynamic setting. ${ }^{1}$ Rather, it is based on physical considerations involving dislocation densities. An extended slip law, which also accounts for statistically stored dislocation densities, is introduced as well. Besides the mechanical displacements, the model of Geers and co-workers is based on the fields of geometrically necessary and statistically stored dislocation densities, which are used to affect crystallographic slip resistance in an extended slip law.

The second model is the one introduced in Ekh et al. [7], which is an extension of the model for isotropic plasticity proposed by Svedberg and Runesson [22]. In [7], a formulation is proposed which models the size effect by assuming that the hardening stress in the yield function depends on both the associated hardening strain (equal to the accumulated plastic slip) and its Hessian along the slip direction similarly to models for isotropic plasticity originally proposed by Aifantis [1]. Similar formulations can be found in Gurtin [13] and Liebe and Steinmann [19]. The thermodynamic setting of Liebe and Steinmann [19] and Ekh et al. [7] is identical. However, in contrast to [19], where the gradient hardening is isotropic, Ekh et al. [7] obtain anisotropic hardening by restricting the gradient effect to each slip direction. Note that a similar strategy, also thermodynamically based, is followed by Vrech and Etse [27] for small strains. Gurtin's formulation [13] is motivated from a so-called microforce balance instead of introducing the

\footnotetext{
${ }^{1}$ One of the reviewer pointed out that there will be an upcoming paper showing that this model can be recasted in a true work-conjugated formulation.
} 
gradient hardening in the free energy.

As a third model, the model of Ekh et al. [7] is in this paper extended by an idea of Lele and Anand [17]. The approach of Lele and Anand [17] is formulated for small strains and isotropic materials, with a similar approach to the one of Ekh et al. [7]. Their idea is to introduce a slip rate with a similar structure to the one of [7]; however, including an additional higher-order dissipative term. Thus, as a third model, we investigate the model of [7] extended by this particular term.

Since we discuss large deformation theories and the field equations are highly coupled, a numerically efficient solution algorithm is needed. A dual mixed finite element algorithm based on [23] is applied to all three models, rendering a coupled two-field problem. The coupling effect is fully taken into account as the system of equations is solved monolithically. The implicit backward Euler scheme is used for the time integration. The resulting system of equations is then solved using a three-level iteration strategy based on a generalized form of domain decomposition, whereby each grain constitutes a subdomain, as suggested in [7]. Due to the fact that each grain can be considered independently (with prescribed boundary conditions), this algorithm is suitable for parallelization.

The main issue of this paper is the comparison of the three models using this algorithm. For all investigated models, it has been shown in previous publications that they are all capable of handling advanced modeling of the hardening. However, no direct comparison has been possible so far, since the discretization approaches and examples presented differ strongly. Such a comparison is desirable in order to further develop micromechanical models capturing size effects in polycrystals. We therefore choose to investigate all three models in the context of anisotropic polycrystals undergoing large deformations.

The paper is organized as follows. In Section 2, the general underlying mathematical framework is introduced. The basic kinematic and constitutive issues and relations of crystal plasticity relevant for all three models are revised. In a second step, in Section 3 the three different models are presented. Having then all constitutive and governing equations at hand, we outline the dual mixed finite element algorithm in Section 4. Subsequently, a two-dimensional polycrystal is investigated as a numerical example in Section 5. The paper ends with a discussion of the different models.

\section{Basic deformation and stress measures}

In this section we reiterate the basic kinematics needed in the following in order to introduce the notation used in this contribution. In large strain plasticity, the main assumption is the classical multiplicative split of the deformation gradient $\boldsymbol{F}$ into an elastic $\boldsymbol{F}^{\mathrm{e}}$ and a plastic part $\boldsymbol{F}^{\mathrm{p}}$ :

$$
\boldsymbol{F}=\boldsymbol{F}^{\mathrm{e}} \cdot \boldsymbol{F}^{\mathrm{p}}
$$

This leads to the following relations for the deformation measures

$$
\overline{\boldsymbol{C}}^{\mathrm{e}}:=\left[\boldsymbol{F}^{\mathrm{e}}\right]^{t} \cdot \boldsymbol{F}^{\mathrm{e}}, \quad \overline{\boldsymbol{E}}^{\mathrm{e}}:=\frac{1}{2}\left(\overline{\boldsymbol{C}}^{\mathrm{e}}-\overline{\boldsymbol{I}}\right),
$$


where the right Cauchy-Green tensor in the intermediate configuration $\overline{\mathcal{B}}$ is denoted by $\overline{\boldsymbol{C}}^{\mathrm{e}}$ and $\overline{\boldsymbol{E}}^{\mathrm{e}}$ is the Green-Lagrange strain.

Next, we recall the stress measures of interest. The 1st Piola-Kirchhoff stress, $\boldsymbol{P}$, and the $2^{\text {nd }}$ Piola-Kirchhoff stress, $\overline{\boldsymbol{S}}^{\mathrm{e}}$ on the intermediate configuration $\overline{\mathcal{B}}$, are defined as:

$$
\boldsymbol{P}:=\boldsymbol{\tau} \cdot \boldsymbol{f}^{t}=\boldsymbol{F}^{\mathrm{e}} \cdot \overline{\boldsymbol{S}}^{\mathrm{e}} \cdot\left[\boldsymbol{f}^{\mathrm{p}}\right]^{t}, \quad \overline{\boldsymbol{S}}^{\mathrm{e}}:=\boldsymbol{f}^{\mathrm{e}} \cdot \boldsymbol{\tau} \cdot\left[\boldsymbol{f}^{\mathrm{e}}\right]^{t},
$$

with the notation $\boldsymbol{f}^{x}:=\left[\boldsymbol{F}^{x}\right]^{-1}$ and $\boldsymbol{\tau}$ being the Kirchhoff stress. Moreover, the Mandel stress, $\overline{\boldsymbol{M}}^{\mathrm{e}}$, on the intermediate configuration $\overline{\mathcal{B}}$, is introduced

$$
\overline{\boldsymbol{M}}^{\mathrm{e}}:=\left[\boldsymbol{F}^{\mathrm{e}}\right]^{t} \cdot \boldsymbol{\tau} \cdot\left[\boldsymbol{f}^{\mathrm{e}}\right]^{t}=\overline{\boldsymbol{C}}^{\mathrm{e}} \cdot \overline{\boldsymbol{S}}^{\mathrm{e}} .
$$

As usual, the model formulation in the context of crystal plasticity is based on the slip-system geometry for each slip-system $\alpha$ : the slip direction $s_{\alpha}$ and the slip plane normal $\boldsymbol{n}_{\alpha}$ which are two orthonormal vectors in the reference configuration $\mathcal{B}_{0}$. For the sake of simplicity, it is assumed that the intermediate configuration is isoclinic such that all slip-systems $\left(\overline{\boldsymbol{s}}_{\alpha}, \overline{\boldsymbol{n}}_{\alpha}\right)$ are fixed in $\overline{\mathcal{B}}$ and known a priori from the given crystal structure. Therefore, the bars are omitted from now on. Then, following Rice [21], the evolution of the plastic deformation is given by the form

$$
\boldsymbol{L}^{\mathrm{p}}=\dot{\boldsymbol{F}}^{\mathrm{p}} \cdot \boldsymbol{f}^{\mathrm{p}}=\sum_{\alpha} \dot{\gamma}_{\alpha}\left[\boldsymbol{s}_{\alpha} \otimes \boldsymbol{n}_{\alpha}\right]
$$

with $\boldsymbol{L}^{\mathrm{p}}$ denoting the plastic velocity gradient tensor. The resolved shear (Schmid) stress $\tau_{\alpha}$, associated with the slip-system $\left(\boldsymbol{s}_{\alpha}, \boldsymbol{n}_{\alpha}\right)$, is defined as

$$
\tau_{\alpha}:=\boldsymbol{s}_{\alpha} \cdot \overline{\boldsymbol{M}}^{\mathrm{e}} \cdot \boldsymbol{n}_{\alpha}=\overline{\boldsymbol{M}}^{\mathrm{e}}:\left[\boldsymbol{s}_{\alpha} \otimes \boldsymbol{n}_{\alpha}\right] .
$$

If $\gamma_{\alpha}$ is interpreted as the slip-system shear, it can be positive or negative and increase or decrease, in which case $\dot{\gamma}_{\alpha} s_{\alpha}=\left|\dot{\gamma}_{\alpha}\right| \operatorname{sign}\left(\dot{\gamma}_{\alpha}\right) s_{\alpha}= \pm\left|\dot{\gamma}_{\alpha}\right| s_{\alpha}$. This motivates to consider both $s_{\alpha}$ and $-s_{\alpha}$ as different slip directions. Henceforth, we follow this idea. $\gamma_{\alpha} \geqslant 0$ can then be interpreted as the accumulated slip-system shear and it is always positive and monotonically-increasing, i.e., $\dot{\gamma}_{\alpha} \geqslant 0$ for all $\alpha=1, \ldots, n$. Of course, from the computational point of view, we have to deal with the expense of doubling the number of slip directions/systems.

\section{Constitutive models}

We assume the St. Venant-Kirchhoff law for the elastic behavior is assumed for all three models. Thus the second Piola-Kirchhoff stress $\overline{\boldsymbol{S}}^{\mathrm{e}}$ on the intermediate configuration is given by

$$
\overline{\boldsymbol{S}}^{\mathrm{e}}=\lambda \overline{\boldsymbol{I}}: \overline{\boldsymbol{E}}^{\mathrm{e}}+2 \mu \overline{\boldsymbol{E}}^{\mathrm{e}}
$$

where $\lambda$ and $\mu$ are the Lamé parameters pertinent to small elastic deformations.

\section{1. $\quad$ Model I}

As a first model, we summarize the one developed by Evers et al. [9], see also [4], which is motivated from physical dislocation densities and evolution equations 
for these. Evers et al. [9] present a model which quantifies for the densities of geometrically necessary dislocations (GNDs) and statistically stored dislocations (SSDs). Only edge dislocation densities are considered as done in $[4]^{1}$. In addition, they assume that all dislocations of interest lie in the slip planes.

The density of GNDs $\rho_{\alpha}^{\text {GND }}$ reads

$$
\rho_{\alpha}^{\mathrm{GND}}=\rho_{\alpha}^{\mathrm{GND}_{0}}-\frac{1}{b} \nabla_{\boldsymbol{X}} \gamma_{\alpha} \cdot \boldsymbol{s}_{\alpha}
$$

where $b$ is magnitude of the Burger's vector and $\rho_{\alpha}^{\mathrm{GND}_{0}}$ denotes the initial GND density.

For SSD densities $\rho_{\alpha}^{\mathrm{SSD}}$, Evers et al. apply a generalization of the rate equation proposed by Essmann and Mughrabi [8]:

$$
\dot{\rho}_{\alpha}^{\mathrm{SSD}}=\frac{1}{b}\left[\frac{1}{L_{\alpha}}-2 y_{c} \rho_{\alpha}^{\mathrm{SSD}}\right]\left|\dot{\gamma}_{\alpha}\right|
$$

The SSD density is initialized by $\rho_{\alpha}^{\mathrm{SSD}}(t=0)=\rho^{\mathrm{SSD}_{0}}>0$ and stays positive. The constant $y_{c}$ denotes the critical annihilation length. The average dislocation segment length of statistically stored dislocations $L_{\alpha}$ can be expressed as

$$
L_{\alpha}=\frac{K}{\sqrt{\sum_{\beta} H_{\alpha \beta}\left|\rho_{\alpha}^{\mathrm{SSD}}\right|+\sum_{\beta} H_{\alpha \beta}\left|\rho_{\alpha}^{\mathrm{GND}}\right|}} .
$$

Following the way of Evers et al. [9], we choose the interaction coefficients $H_{\alpha \beta}=$ $h_{0} \forall \alpha, \beta$ with $h_{0}$ being the constant immobilization coefficient. The evolution relation for the slip-system shear $\gamma_{\alpha}$ is given by a power law

$$
\dot{\gamma}_{\alpha}=\dot{\gamma}_{0}\left[\frac{\left|\tau_{\alpha}^{\mathrm{eff}}\right|}{s_{\alpha}}\right]^{m}
$$

relating the slip rates $\dot{\gamma}_{\alpha}$ to the effective shear stress $\tau_{\alpha}^{\text {eff }}$ and the slip-system strength $s_{\alpha}$. The plastic shear rate $\dot{\gamma}_{0}$ and the rate sensitivity parameter $m$ are constant material parameters. The effective shear stress $\tau_{\alpha}^{\text {eff }}$ is defined as

$$
\tau_{\alpha}^{\mathrm{eff}}=\tau_{\alpha}-\overline{\boldsymbol{\tau}}^{b}:\left[\boldsymbol{s}_{\alpha} \otimes \boldsymbol{n}_{\alpha}\right]
$$

where $\bar{\tau}^{b}$ is the global back-stress tensor

$$
\overline{\boldsymbol{\tau}}^{b}=\sum_{\alpha} \tau_{\alpha}^{e}\left[\left[\boldsymbol{s}_{\alpha} \otimes \boldsymbol{n}_{\alpha}\right]+\left[\boldsymbol{s}_{\alpha} \otimes \boldsymbol{n}_{\alpha}\right]^{t}\right]
$$

The resulting shear stress $\tau_{\alpha}^{e}$

$$
\tau_{\alpha}^{e}=\frac{\mu b R^{2}}{8[1-\nu]} \nabla_{\boldsymbol{X}} \rho_{\alpha}^{\mathrm{GND}} \cdot \boldsymbol{s}_{\alpha}
$$

\footnotetext{
${ }^{1}$ The model introduced in [9] is formulated for edge and screw dislocation densities. However, the numerical example presented in [9] is restricted to self-hardening and double slip where only edge dislocations are considered.
} 
develops independently. The circular region around a material point $\boldsymbol{X}$ with radius $R$ accounts for the geometrically necessary dislocations contributing to the shear stress on slip-system $\alpha$. Moreover, $\mu$ is the shear modulus and $\nu$ is Poisson's ratio. The slip-system strength $s_{\alpha}$ reads

$$
s_{\alpha}=c \mu b \sqrt{\sum_{\beta} a_{0}\left|\rho_{\beta}^{\mathrm{SSD}}\right|+\sum_{\beta} a_{0}\left|\rho_{\beta}^{\mathrm{GND}}\right|},
$$

where $c$ is a constant material parameter. In particular, it is a function of the SSD and GND densities.

\section{2. $\quad$ Model II}

As a second model, we investigate the approach introduced by Ekh et al. [7] which is summarized below. In contrast to model I, it is embedded in a thermodynamic framework. Moreover, it is simpler with respect to hardening, but still showed realistic simulation results in [7]. The free energy $\bar{\Psi}_{0}$ per unit intermediate volume is introduced as a function of the intermediate Green-Lagrange strain $\overline{\boldsymbol{E}}^{\mathrm{e}}$, the slip-system shears $\gamma_{\alpha}$ and its gradient $\nabla_{\boldsymbol{X}} \gamma_{\alpha}$ :

$$
\begin{aligned}
\bar{\Psi}_{0}\left(\overline{\boldsymbol{E}}^{\mathrm{e}},\left\{\gamma_{\alpha}\right\},\left\{\nabla_{\boldsymbol{X}} \gamma_{\alpha}\right\}\right)= & \frac{\lambda}{2}\left[\operatorname{tr} \overline{\boldsymbol{E}}^{\mathrm{e}}\right]^{2}+\mu \operatorname{tr}\left(\left(\overline{\boldsymbol{E}}^{\mathrm{e}}\right)^{2}\right) \\
& +\frac{1}{2} \sum_{\alpha} H_{\alpha}^{1} \gamma_{\alpha}^{2}+\frac{1}{2} \sum_{\alpha} l_{\alpha}^{2} \nabla_{\boldsymbol{X}} \gamma_{\alpha} \cdot\left[H_{\alpha}^{g} \boldsymbol{s}_{\alpha} \otimes \boldsymbol{s}_{\alpha}\right] \cdot \nabla_{\boldsymbol{X}} \gamma_{\alpha}(1)
\end{aligned}
$$

It is additively decomposed in a hyperelastic contribution following the St. Venant model and a hardening contribution. The hardening contribution to the free energy can be decomposed further into contributions from local and gradient hardening, respectively. The corresponding hardening moduli, $H_{\alpha}^{l}$ and $H_{\alpha}^{g}$, are chosen as constant and positive semi-definite measures associated with each slip-system $\alpha$.

This leads to the following hardening stresses $\kappa_{\alpha}$ which are defined in such a way that they can be derived from the free energy via

$$
\begin{array}{cc}
\kappa_{\alpha}:=\frac{\partial \bar{\Psi}_{0}}{\partial \gamma_{\alpha}}-\operatorname{Div}\left(\frac{\partial \bar{\Psi}_{0}}{\partial\left(\nabla_{\boldsymbol{X}} \gamma_{\alpha}\right)}\right) \quad \text { in } \mathcal{B}_{0, \text { grain }}, \alpha=1,2, \ldots, n_{\text {slip }} \\
\kappa_{\alpha}^{(b)}:=\quad \quad \quad \quad \quad \frac{\partial \bar{\Psi}_{0}}{\partial\left(\nabla_{\boldsymbol{X}} \gamma_{\alpha}\right)} \quad \text { on } \partial \mathcal{B}_{0, \text { grain }, \alpha=1,2, \ldots, n_{\text {slip }}}
\end{array}
$$

see Appendix for further details. Here, $\boldsymbol{N}$ is the outward unit normal to the boundary $\partial \mathcal{B}_{0 \text {, grain }}$ and the superscript " $b$ " denotes "boundary".

The evolution of the plastic slip $\gamma_{\alpha}$ is assumed to follow a viscoplastic power law

$$
\dot{\gamma}_{\alpha}=\frac{1}{t_{*}}\left[\frac{<\tau_{\alpha}-Y_{\alpha}-\kappa_{\alpha}>}{C_{0}}\right]^{m},
$$

with $\langle x\rangle:=1 / 2[x+|x|]$ denoting the Macaulay brackets. The material parameter $C_{0}$ is constant and the same for all slip-systems and $Y_{\alpha}$ and $t_{*}$ denote the initial yield stress and the relaxation time, respectively. 


\subsection{Model III}

Following Gurtin [14], Lele and Anand [17] propose a thermodynamic-consistent small-deformation strain-gradient theory for isotropic viscoplastic materials similar to the large-deformation model II (which is formulated for anisotropic materials). As in the case of model II, the free energy is split additively into an elastic and a plastic contribution and the latter depends on the slip-system shear gradient $\nabla_{\boldsymbol{X}} \gamma_{\alpha}$. Moreover, the free energy $\bar{\Psi}_{0}$ acts an potential for what they call the energetic microstress $\boldsymbol{\xi}_{\alpha}^{\text {en }}$ - in a similar way to the gradient hardening stress $\kappa_{\alpha}$ of Ekh et al. [7]. In addition to the approach of Ekh et al. [7], they introduce a further length scale, the constant dissipative lengthscale $l_{d}$, corresponding to dissipative effects associated with the slip rate gradient $\nabla_{\boldsymbol{X}} \dot{\gamma}_{\alpha}$. Since promising results are presented in [17] for the case of small deformation and isotropy, we extend the flow rule of the large deformation, anisotropic model II in the spirit of Anand, Gurtin and Lele and propose the following expression as the flow rule of model III

$$
\dot{\gamma}_{\alpha}=\frac{1}{t_{*}}\left[\frac{<\tau_{\alpha}-Y_{\alpha}-\kappa_{\alpha}>}{C_{0}}\right]^{m}+l_{d}^{2}{ }^{0} s_{\alpha} \operatorname{Div}\left(\left[\frac{d_{\alpha}}{{ }^{0} d}\right]^{q} \frac{\nabla_{\boldsymbol{X}} \dot{\gamma}_{\alpha} \cdot \boldsymbol{s}_{\alpha}}{d_{\alpha}} \boldsymbol{s}_{\alpha}\right)
$$

Hence, the last term in Eq. (20) is nonstandard. Interest in such a higher-order dissipative term has arisen only recently, see e.g. the works of [12], [14], and the very recent [17]. Here, the scalar ${ }^{0} s_{\alpha}=s_{\alpha}(t=0)>0$ represents the initial flow resistance, see also Eq. (16). Furthermore, ${ }^{0} d$ is a reference rate and $d_{\alpha}$ denotes the scalar non-negative effective flow-rate

$$
d_{\alpha}:=\sqrt{\left|\dot{\gamma}_{\alpha}\right|^{2}+l_{d}^{2}\left|\nabla_{\boldsymbol{X}} \dot{\gamma}_{\alpha}\right|^{2}}
$$

\subsection{Model comparison}

Here it is appropriate to point out the similarities of the three models. Taking another look at the rate equations of the slip-system shear of model I and II, i.e., Eqs. (12) and (19), highlights the differences of those two approaches. We rewrite the rate equation of model I by inserting the effective shear stress relations (13)(15) into the flow rule of model I (Eq. (12)) first. Subsequently, the GND density $\rho_{\alpha}^{\mathrm{GND}}$ is substituted by relation (9). Consequently, we obtain:

$$
\begin{aligned}
\dot{\gamma}_{\alpha} & \left.=\dot{\gamma}_{0}\left[\frac{\left.\left|\tau_{\alpha}+\frac{\mu R^{2}}{8[1-\nu]}\left[\sum_{\beta} \boldsymbol{\nabla}_{\boldsymbol{X}}\left[\boldsymbol{\nabla}_{\boldsymbol{X}} \gamma_{\beta} \cdot \boldsymbol{s}_{\beta}\right] \cdot \boldsymbol{s}_{\beta} P_{\beta \alpha}\right]\right|\right]^{m}}{s_{\alpha}}\right]^{m}\right]^{m}, \\
& =\dot{\gamma}_{0}\left[\frac{\left|\tau_{\alpha}+\frac{\mu R^{2}}{8[1-\nu]}\left[\sum_{\beta} P_{\beta \alpha}\left[\boldsymbol{s}_{\beta} \otimes \boldsymbol{s}_{\beta}\right]:\left[\boldsymbol{\nabla}_{\boldsymbol{X}} \otimes \boldsymbol{\nabla}_{\boldsymbol{X}}\right] \gamma_{\beta}\right]\right|}{s_{\alpha}},\right.
\end{aligned}
$$

where we define the scalar quantity $P_{\beta \alpha}=\left[\left[\boldsymbol{s}_{\beta} \otimes \boldsymbol{n}_{\beta}\right]+\left[\boldsymbol{s}_{\beta} \otimes \boldsymbol{n}_{\beta}\right]^{t}\right]:\left[\boldsymbol{s}_{\alpha} \otimes \boldsymbol{n}_{\alpha}\right]$ which accounts for cross-gradient hardening. Analogously, inserting the hardening stress relation (18) into the flow rule of model II, i.e., Eq. (19), yields

$$
\dot{\gamma}_{\alpha}=\frac{1}{t_{*}}\left[\frac{<\tau_{\alpha}-Y_{\alpha}-H_{\alpha}^{l} \gamma_{\alpha}+l_{\alpha}^{2} H_{\alpha}^{g}\left[\boldsymbol{s}_{\alpha} \otimes \boldsymbol{s}_{\alpha}\right]:\left[\boldsymbol{\nabla}_{\boldsymbol{X}} \otimes \boldsymbol{\nabla}_{\boldsymbol{X}}\right] \gamma_{\alpha}>}{C_{0}}\right]^{m} .
$$


Clearly, in both cases, the rate equations contain terms depending on the second spatial derivatives of the slips, i.e., $\left[\nabla_{\boldsymbol{X}} \otimes \nabla_{\boldsymbol{X}}\right] \gamma_{\alpha}$. These terms influence the effective resolved stress driving the glide-system dislocation motion. ${ }^{1}$

Evers et al. [9] use the absolute signs in the numerator whereas Ekh et al. [7] employ the Macaulay brackets. Therefore, the rate of the plastic slip can be equal to zero in case of model II as opposed to model I where it is always positive. Thus, in case of model I the slip systems are activated viscously at the stress of $s_{\alpha}$. On the other hand, model II discretely activates the slip systems at the yield stress $Y_{\alpha}$.

One can easily identify what is called the reference plastic strain rate $\dot{\gamma}_{0}$ in [9] with the inverse of the relaxation time $t_{*}$ in [7]. Moreover, the material parameter $C_{0}$ of model II has the unit of a stress and plays the same role the slip-system strength $s_{\alpha}$ does in the slip-system rate equation of model I. However, $C_{0}$ is chosen to be constant and the same for all slip-systems $\alpha$. On the contrary, the slip-system strength differs for slip-systems and depends on the SSD and GND densities and consequently is an implicit function of the slip-system shear as well, i.e., $s_{\alpha}=s_{\alpha}\left(\rho^{\mathrm{SSD}}, \rho^{\mathrm{GND}} ; \gamma\right)$. Anticipating the material parameters stated in Table 1 reveals that in the simplest case possible, i.e., no slip and no GND density at initial time $t=0$, both are of approximately the same magnitude $\left(1[\mathrm{MPa}]\right.$ vs. $3.7[\mathrm{MPa}]$ for $C_{0}$ respectively $\left.{ }^{0} s_{\alpha}=s_{\alpha}(t=0)=c \mu b \sqrt{a_{0} \rho_{\alpha}^{\mathrm{SSD}_{0}}}\right)$.

While model I accounts for local hardening via the fact that the denominator, the slip-system strength $s_{\alpha}$, depends a.o. on the square root of the SSD densities $\rho^{\mathrm{SSD}}$, Ekh et al. [7] include a local hardening contribution in the free energy function $\bar{\Psi}_{0}$ and therefore in the hardening stresses $\kappa_{\alpha}$ - which is part of the numerator. Furthermore, both approaches account for gradient hardening in a similar, but not the same way, via directional gradients of the slip-system shear along the given slip directions via $\nabla_{\boldsymbol{X}} \gamma_{\alpha} \cdot \boldsymbol{s}_{\alpha}$.

Lele and Anand's approach [17] is based on the so-called microforce balance representing a viscoplastic yield condition

$$
\tau_{\alpha}+\operatorname{Div}\left(\boldsymbol{\xi}_{\alpha}^{\mathrm{en}}+\boldsymbol{\xi}_{\alpha}^{\mathrm{diss}}\right)=f_{\alpha}\left(\gamma_{\alpha}, \dot{\gamma}_{\alpha}\right)
$$

see also Gurtin and Anand [14]. Here, $\boldsymbol{\xi}_{\alpha}^{\text {en }}$ and $\boldsymbol{\xi}_{\alpha}^{\text {diss }}$ are referred to as the energetic and the dissipative microstress, respectively, and they expend power over the sliprate gradient $\nabla_{\boldsymbol{X}} \dot{\gamma}_{\alpha}$. What is here denoted by a function $f$ is referred to as a scalar microscopic stress in [17] which expends power over the slip-rate $\dot{\gamma}_{\alpha}$. In case of model III, the energetic and the dissipative microstress are given by the relations

$$
\begin{aligned}
\boldsymbol{\xi}_{\alpha}^{\mathrm{en}} & =l_{\alpha}^{2} H_{\alpha}^{g} \boldsymbol{s}_{\alpha}\left[\boldsymbol{s}_{\alpha} \cdot \nabla_{\boldsymbol{X}} \gamma_{\alpha}\right] \\
\boldsymbol{\xi}_{\alpha}^{\text {diss }} & =l_{d}^{2}{ }^{0} s_{\alpha}\left[\frac{d_{\alpha}}{{ }^{0} d}\right]^{q} \frac{\boldsymbol{\nabla}_{\boldsymbol{X}} \dot{\gamma}_{\alpha} \cdot \boldsymbol{s}_{\alpha}}{d_{\alpha}} \boldsymbol{s}_{\alpha} .
\end{aligned}
$$

Moreover,

$$
f_{\alpha}\left(\gamma_{\alpha}, \dot{\gamma}_{\alpha}\right)=\left[t_{*} \dot{\gamma}_{\alpha}\right]^{1 / m} C_{0}+Y_{\alpha}+H_{\alpha}^{l} \gamma_{\alpha}
$$

\footnotetext{
${ }^{1}$ Different stress terms and their relations are compared in [11] - with the main focus on linking the phenomenological Cosserat theory, the statistical theory of dislocations and strain gradient crystal plasticity.
} 
Clearly, the energetic microstress $\boldsymbol{\xi}_{\alpha}^{\text {en }}$ and the dissipative microstress $\boldsymbol{\xi}_{\alpha}^{\text {diss }}$ are tangent to the corresponding slip plane $\alpha$. If plastic loading is obtained in case of model II, i.e., if $\tau_{\alpha}-Y_{\alpha}-\kappa_{\alpha}>0$, the microforce balance is obtained with

$$
\begin{aligned}
\boldsymbol{\xi}_{\alpha}^{\mathrm{en}} & =l_{\alpha}^{2} H_{\alpha}^{g} \boldsymbol{s}_{\alpha}\left[\boldsymbol{s}_{\alpha} \cdot \nabla_{\boldsymbol{X}} \gamma_{\alpha}\right] \\
\boldsymbol{\xi}_{\alpha}^{\text {diss }} & =\mathbf{0} \\
f_{\alpha}\left(\gamma_{\alpha}, \dot{\gamma}_{\alpha}\right) & =\left[t_{*} \dot{\gamma}_{\alpha}\right]^{1 / m} C_{0}+Y_{\alpha}+H_{\alpha}^{l} \gamma_{\alpha} .
\end{aligned}
$$

1

In a similar way, in the case of plastic loading $\tau_{\alpha}^{\text {eff }}>0$, the rate equation (12) of model I could be interpreted as a generalized microforce balance with the following choices:

$$
\begin{aligned}
\boldsymbol{\xi}_{\alpha}^{\mathrm{en}} & =\frac{\mu R^{2}}{8[1-\nu]} \sum_{\beta} P_{\beta \alpha} \boldsymbol{s}_{\beta}\left[\boldsymbol{s}_{\beta} \cdot \nabla_{\boldsymbol{X}} \gamma_{\beta}\right], \\
\boldsymbol{\xi}_{\alpha}^{\text {diss }} & =\mathbf{0} \\
f_{\alpha}\left(\gamma_{\alpha}, \dot{\gamma}_{\alpha}, \nabla_{\boldsymbol{X}} \gamma_{\alpha}\right) & =\left[\frac{\dot{\gamma}_{\alpha}}{\dot{\gamma}_{0}}\right]^{1 / m} s_{\alpha} .
\end{aligned}
$$

We choose a more generalized comparison at this point, because the slip strength $s_{\alpha}$ depends on the slip rate $\dot{\gamma}_{\alpha}$ as well as on the slip gradient $\boldsymbol{\nabla}_{\boldsymbol{X}} \gamma_{\alpha}$. Moreover, in the thermodynamic setting of [17], the energetic microstresses $\boldsymbol{\xi}_{\alpha}^{\text {en }}$ are determined in terms of the free energy acting as a potential.

\section{Numerical framework}

\subsection{Governing equations: strong and weak formulation}

The mechanical problem is governed by the quasi-static balance of momentum

$$
\mathbf{0}=\operatorname{Div} \boldsymbol{P}+\boldsymbol{b}_{v}
$$

with $\boldsymbol{b}_{v}$ representing the volume forces.

For the slip problem, we apply the following procedure for all three models: In the spirit of Svedberg and Runesson [23], we introduce an auxiliary scalar field $g_{\alpha}$ for the directional gradient of the slip-system shear $\gamma_{\alpha}$ along the given slip direction $s_{\alpha}$,

$$
g_{\alpha}:=\nabla_{\mathbf{X}} \gamma_{\alpha} \cdot s_{\alpha}
$$

Moreover, following [23], we approximate the slip-system shear $\gamma_{\alpha}$ and the scalar $g_{\alpha}$ independently in $\mathcal{B}_{0 \text {, grain }}$ using a so-called dual mixed space-variational format of the gradient equation $g_{\alpha}-\nabla_{\boldsymbol{X}} \gamma_{\alpha} \cdot \boldsymbol{s}_{\alpha}=0$, which is a global equation in space.

\footnotetext{
${ }^{1}$ As Macaulay brackets have been used in the formulation of the flow rule for model II and III, the microforces are determinate because we assume microclamped respectively microfree grain boundary conditions (cf. Eq. (31)). In case of an elastically deforming grain with a plastically deforming neighboring grain, the microforces at its grain boundary might be indeterminate - e.g. if continuity conditions are assumed at the grain boundary.
} 
In particular, a three-level Newton-Raphson iteration scheme will be applied, see Section 4.2, in which it is possible to choose the FE-approximation of the gradient $g_{\alpha}$ as $\mathcal{P}_{q}$ (piecewise polynomial of order $q$ ), whereas the FE-approximation of the slip $\gamma_{\alpha}$ is chosen as $\mathcal{P}_{q-1}$.

$$
\begin{aligned}
& \text { We assume "microclamped" boundary conditions }{ }^{1} \text {, i.e., } \\
& \qquad \gamma_{\alpha}=0 \text { on } \partial \mathcal{B}_{0 \text {, grain. }}
\end{aligned}
$$

Remark: For a quite detailed description of the finite element discretization, the reader is referred to [7], in which the exact FE-approximations, the matrix formulation of the finite element problem, the algorithmic iteration matrices and tool boxes describing the flow of the algorithm are given.

\subsection{Three-level Newton-Raphson iteration strategy}

The basic solution strategy is Newton-Raphson iterations on three levels. Global $\mathrm{FE}$-solutions of the balance of momentum and gradient equations will yield the displacement field $\boldsymbol{u}(\boldsymbol{X})$ and the gradient field $g_{\alpha}(\boldsymbol{X})$ at $t=t_{n}$. There exist different model assumptions on "geometric constraints" on the grain structure, which infer restrictions on the displacement $\boldsymbol{u}$. It is highly desirable to have a displacement field as unconstrained as possible. Therefore, we only prescribe the displacements at the grain-structure (RVE) boundary. As part of this strategy, we solve for the displacements on the grain boundaries in an outer iteration loop called "grain boundary iteration loop". Then, in the "inner grain iteration loop", updated values of displacements $\boldsymbol{u}$ and gradients $g_{\alpha}$ within each grain are computed. During the "inner grain iteration loop", the displacements on the grain boundaries are kept fixed, given the updated values from the "grain boundary iteration loop". Therefore, the resulting algorithm is suitable for parallelization.

Furthermore, a third iteration loop is carried out. The purpose of this "local iteration loop" is to find updated values for the slip $\gamma_{\alpha}$ (and in case of model I also for the SSD density $\rho_{\alpha}^{\mathrm{SSD}}$ ) in each Gauss point, given values of $\boldsymbol{u}$ and $\underline{g}$ (as provided from the outer loop).

As mentioned above, see Section 4.1, the intergrain conditions are assumed to be micro-clamped, i.e., there is no explicit coupling of slip gradients across the grain boundaries. Consequently, we do not have to solve for the gradients $g_{\alpha}$ during the "grain boundary iteration loop". In the grain iteration loop, the fully discretized system of equations are coupled. Furthermore, in the inner iteration loop of model $\mathrm{I}$, the rate equations (12) and (10) are coupled. In both cases the coupled system is solved monolithically.

\footnotetext{
${ }^{1}$ Both, microclamped and microfree boundary conditions, rule out the possibility of interaction of slip systems across a grain boundary and is therefore quite restrictive. Nevertheless, it is quite popular and has been used by e.g. [7, 13, 14, 17, 19, 26]. There also exist contributions introducing boundary conditions that may account for grain interactions, see e.g. [12]. In this contribution we limit our analysis to the particular case described above, as the influence of different grain boundary conditions is part of future research and a topic of its own.
} 


\subsection{Time integration}

Although the governing equations (29) and (30) are not time-dependent, on the "local iteration loop" level the model contains evolution equations which have to be discretized in time. The state at $t=t_{n-1}$ is assumed to be known for a given time history of the pertinent loading. Thus, the fully implicit backward Euler scheme is applied to Eqs. (6), (10), (12), (19) and (20). For example, in case of the velocity gradient $\boldsymbol{L}^{\mathrm{p}}=\dot{\boldsymbol{F}}^{\mathrm{p}} \cdot \boldsymbol{f}^{\mathrm{p}}$, i.e., Eq. (6), this leads to

$$
\boldsymbol{I}-{ }^{n-1} \boldsymbol{F}^{\mathrm{p}} \cdot{ }^{n} \boldsymbol{f}^{\mathrm{p}}-\sum_{\alpha} \Delta t \dot{\gamma}_{\alpha}\left[\boldsymbol{s}_{\alpha} \otimes \boldsymbol{m}_{\alpha}\right]=\mathbf{0} .
$$

Here, $\Delta t=\left[t_{n}-t_{n-1}\right]$ denotes the current time step and we initialize ${ }^{0} \boldsymbol{f}^{\mathrm{p}}=\boldsymbol{I}$ and ${ }^{0} \gamma_{\alpha}=0$.

Furthermore, the slip-system shear rate equations have to discretized in time for all three models. For the sake of comprehensibility, we leave the spatial discretization aside for this moment. Therefore, the semi-discrete formulations of Eqs. (12), (19) and (20) read:

model I

$$
0=\frac{1}{\dot{\gamma}_{0}} \Delta \gamma_{\alpha}-\Delta t\left[\frac{\left|\tau_{\alpha}^{\mathrm{eff}}\right|}{s_{\alpha}}\right]^{m}
$$

model II

$$
0=t_{*} \Delta \gamma_{\alpha}-\Delta t\left[\frac{<\tau_{\alpha}-Y_{\alpha}-\kappa_{\alpha}>}{C_{0}}\right]^{m},
$$

model III

$$
0=t_{*} \Delta \gamma_{\alpha}-\Delta t\left[\frac{<\tau_{\alpha}-Y_{\alpha}-\kappa_{\alpha}>}{C_{0}}\right]^{m}-l_{d}^{20} s_{\alpha} \operatorname{Div}\left(\left[\frac{d_{\alpha}}{{ }^{0} d}\right]^{q} \frac{\Delta g_{\alpha}}{d_{\alpha}} \cdot s_{\alpha}\right) .
$$

In case of the rate equation of model III, we used $\nabla_{\boldsymbol{X}} \dot{\gamma}_{\alpha} \cdot \boldsymbol{s}_{\alpha}=\dot{g}_{\alpha}$.

\section{Numerical example: macro-scale simple shear}

\subsection{Set-up of the model}

The grain-structure, occupying the square domain $\mathcal{B}_{\square}$ in $2 \mathrm{D}$ with boundary $\partial \mathcal{B}_{\square}$, as shown in Figure 1, is considered. Dirichlet boundary conditions on $\partial \mathcal{B}_{\square, \mathrm{D}}$ are chosen that correspond to a prescribed macro-scale deformation gradient $\boldsymbol{F}_{m}=\boldsymbol{I}+\boldsymbol{H}_{m}$ (where the subscript " $m$ " indicates a macro-scale quantity). Hence, the boundary conditions for the mechanical problem are

$$
\boldsymbol{u}(\boldsymbol{X})=\boldsymbol{H}_{m} \cdot \boldsymbol{X}, \quad \boldsymbol{X} \in \partial \mathcal{B}_{\square}
$$

As a consequence, the appropriate spaces of displacements for the sub-scale are defined as

$$
\mathbb{U}\left(\boldsymbol{H}_{m}\right)=\left\{\boldsymbol{u} \mid \boldsymbol{u}=\boldsymbol{H}_{m} \cdot \boldsymbol{X} \text { for } \boldsymbol{X} \in \partial \mathcal{B}_{\square}\right\}
$$

The grain-structure consists of 4 grains, see Figure 1, and is discretized by 1990 elements (1384 nodes). 
So far, nothing has been said about the number of slip-systems. Henceforth, we limit the numerical study to double slip in order reduce computation time. From the modeling point of view this means $\alpha=4$, as explained at the end of Section 2. The directions $\boldsymbol{s}_{\alpha}$ of the slips are randomly distributed, see Figure 1. Values of the material parameters are listed in Table 1.

Plane strain is assumed. In particular, we consider the standard representative example of simple shear on the macro-scale in order to highlight the major characteristics of the different models. Simple shear is defined by $\overline{\boldsymbol{F}}=\boldsymbol{I}+\bar{\gamma} \boldsymbol{e}_{1} \otimes \boldsymbol{e}_{2}$, where $\bar{\gamma}$ is the macroscopic shear deformation. Computations are carried out in monotonic loading with $\bar{\gamma}_{\max }=0.15$ with the constant loading rate of $0.03[1 / \mathrm{s}]$.

Remark (Material parameters): The slip rate equation of model I, i.e., Eq. (12), is defined in terms of the reference plastic strain rate $\dot{\gamma}_{0}$ and the slip resistance $s_{\alpha}$. In contrast, the slip rate equations of model II and III, i.e., Eqs. (19) and (20), are expressed in terms of the relaxation time $t_{*}$ and the constant $C_{0}$. In order to derive comparable results, one has to take care that $1 /\left[t_{*} C_{0}^{m}\right] \approx \dot{\gamma}_{0} / s_{\alpha}^{m}(t=0)$. In addition, the fraction $\mu R /[8[1-\nu]]$ in Eq. (22) (model I) plays a similar role as $l_{\alpha}^{2} H_{\alpha}^{g}$ in Eq. (23) (model II).

As to the choice of the yield stress, a constant value of $Y_{\alpha}$ is chosen in model II, whereas the yield stress of model I is strain-rate dependent and incorporated indirectly via $s_{\alpha}$.

\subsection{Discussion of results}

First, model I is examined. Figure 2 shows a size effect for fixed material parameters but for increasing side length $L$ of the grain-structure. The plot shows a representation of the plastic strain field in terms of "effective" effective shear strain $\gamma_{\text {eff }} \stackrel{\text { def }}{=} \sqrt{\gamma_{1}^{2}+\gamma_{2}^{2}+\gamma_{3}^{2}+\gamma_{4}^{2}}$. Since the (absolute) size of the boundary layers with large gradient effects should be approximately the same regardless of the actual size of the grains, the boundary layers will appear thinner when the grain size increases (see Figure 2).

The macroscopic stress-strain response in terms of the nominal shear stress component $\bar{P}_{12}$ versus the macroscopic shear $\bar{\gamma}$ is shown in Figure 3 . As expected, stiffer response in the hardening regime can be seen for smaller grain size. This observation is in agreement with the findings of Bayley et al. [4] and Evers et al. [9]. The size-dependence of the stress-strain response is due the existence of the slip gradient in the numerator of Eq. (22). As already reported by Evers et al. [9], a size dependence for both densities can be found. Figure 4 exemplarily shows the distribution of the GND density $\rho_{\text {GND }}$ for two sample lengths. On the left $L=4 \mu \mathrm{m}$ is depicted and on the right the distribution for a grain-structure with length $L=16 \mu \mathrm{m}$ is plotted. In agreement with the results of $[9,17]$, the GND density $\rho_{\mathrm{GND}}$ mainly develops near the grain boundaries. Moreover, we also observe the significant difference between the results for the two lengths. This is due to the fact that the distance between the grain middle and the boundary is much smaller in the case of $L=4 \mu \mathrm{m}$ - the boundary being the place where the plastic slip is blocked and therefore GNDs develop. On the contrary, the SSD density $\rho_{\mathrm{SSD}}$ remains small near the grain boundaries, see Figure 5. Furthermore, the order of magnitude of the GND and the SSD denisties are roughly the same. For the sake of brevity and as we mainly aim at comparing different approaches, we omit figures of this finding for 
the other examples simulated with model I and instead refer to the discussion in [9].

One of the differences between model I and II is the use of the absolute signs respectively Macauley brackets. We ran simulations in which we applied Macauley brackets to Eq. (12). Due to the fact that load is applied only in one direction, there is no difference for this particular numerical example. However, if loading directions change during the simulation, there should be an impact.

In order to examine the effect of cross-hardening only, simulations are carried out with a constant slip resistance $s_{\alpha}=C_{0}=1[\mathrm{MPa}]$. In other words, local hardening is omitted. The results are depicted in Figures 6 and 7. Due to the gradient contribution, the stress-strain response still depends on the grain-structure side length $L$. However, the material response is significantly softer than in Figure 3.

In [9], the rate sensitivity parameter $m=20$ is used, which is significantly lower than our choice. Therefore, simulations with a rate sensitivity parameter of $m=5$ are run as well in order to investigate its influence, see Figure 8. The higher the rate sensitivy parameter $m$, the softer responds the material.

Now, model II is studied. In all simulations of model II, the rate sensitivity parameter $m=1$ is used. Figure 9 presents the distribution of the effective plastic slip $\gamma_{\text {eff }}$ according to Eq. (19) with an initial yield stress $Y_{\alpha}=10^{3}[\mathrm{MPa}]$ for different grain-structure side lengths. The macroscopic stress-strain response is shown in Figure 10. The results are qualitatively similar to those reported in [7].

As in the case of model I, the size-dependent stress-strain response is due to the gradient effect. Although both models reach a similar macroscopic stress $\bar{P}_{12}$ at $15 \%$ shear, the effective slip-system shear of model I is smaller (cf. Figures 2 and 9). For the chosen parameter values less plastic strain develops. Thus, model I accommodates more shear elastically than model II.

In order to investigate the influence of the initial yield stress $Y_{\alpha}$ and the local hardening module $H_{\alpha}^{l}$ (which contributes to the hardening stresses $\kappa_{\alpha}$ ) in model II, those parameters are neglected one after the other. Figures 11 and 12 illustrate the solution with initial yield stress $Y_{\alpha}=0[\mathrm{MPa}]$ and the local hardening module $H_{\alpha}^{l}=10^{4}[\mathrm{MPa}]$ for all slip-systems $\alpha=1, \ldots, 4$. As expected, the plots of the effective hardening strain $\gamma_{\text {eff }}$ (Figure 11) and the macroscopic stress-strain response (Figure 12) reveal that the initial yield stress $Y_{\alpha}=10^{3}[\mathrm{MPa}]$ leads to plastic yielding from the beginning of the deformation.

Subsequently, the local hardening module is additionally chosen to be zero, i.e., $H_{\alpha}^{l}=0[\mathrm{MPa}]$ for all slip-systems $\alpha=1, \ldots, 4$ and $Y_{\alpha}=0[\mathrm{MPa}]$. It is observed that a material with a vanishing local hardening module $H_{\alpha}^{l}$ still shows a size-dependent stiffness response, see Figures 13 and 14. The general stiffness of the material decreases considerably, though. As already mentioned in Section 3.4, model II includes the local hardening contribution in the hardening stress $\kappa_{\alpha}$ and, thus, in the numerator, whereas model I takes it into account via the slip-system strength $s_{\alpha}$ and, therefore, in the denominator. The comparison of Figures 3 and 7 versus 12 and 14 underlines that, nevertheless, both models have a similar local hardening response. Figures 3 and 12 include local hardening, whereas Figures 7 and 14 neglect this contribution - leading to a significant decrease of the material's stiffness. Moreover, the cases studied in Figures 6 and 7 respectively 13 and 14 
show the influence of cross-hardening and no cross-hardening. In this special case, the only difference between the two slip laws of model I and II is that cross-hardening is included in the gradient hardening of model I.

In all three cases, the macroscopic stress-strain response is grain-structure size dependent. However, there is no size-dependence in the initial yield limit, see Figures 10, 12 and 14. This contradicts the well-known Hall-Petch relation which states that the yield limit is in fact size dependent.

Model II corresponds to what Anand, Lele and Gurtin $[14,17]$ call the case of gradient energetics respectively energetic hardening. Combining model II with what they refer to as "dissipative strengthening" leads to model III. The results for model III are pointed out in Figures 15 - 17. As stated by Lele and Anand [17], the divergence term in the flow rule (20) leads to a size-dependent initial yield limit, see Figure 17. However, for the parameters chosen in this example of large deformations, Figure 17 shows that the effect unfortunately is not strong as in the example of [17]. However, in [17] in all the simulations in which the nonstandard higher-order dissipative term was considered, the energetic hardening and standard isotropic hardening were set at zero. In our example, a higher value for the dissipative lengthscale $l_{d}$ would have been desirable in order to receive a considerable side-length dependent yield stress for the case that local hardening is admitted (Figure 16).

As stated in [17], the model "possesses a mathematically attractive structure, our experience with numerical experiments which use these constitutive equations is that they are too tightly coupled". A completely different numerical approach was applied in this contribution ${ }^{1}$

, nevertheless and unfortunately, for larger $l_{d}$ the code did not converge.

\section{Conclusion}

Three different frameworks for including gradient hardening into crystal plasticity models were studied. Model I is physically based and has already proven to be capable of handling advanced modeling of hardening. As compared to this model, the purpose of model II is to be simple, e.g. with respect to the hardening, but still able to show realistic simulation results. Moreover, it is formulated in a thermodynamic setting - as is model III. In both cases, the inelastic part of the free energy was assumed to include contributions from the gradient of hardening along each slip direction.

One can clearly see the different behavior depending on the model assumptions. Model I is capable of mapping more effects than model II, such as cross-hardening. However, it is also much more complex which leads to higher computation time. Model III is a first step towards mapping the Hall-Petch relation, i.e. the squareroot dependence of the yield strength on the grain-size. Unfortunately, numerical convergence problems so far limit the advantage of the mathematically attractive structure. By investigating the meaning and the influence of some of the parameters of the strain gradient crystal plasticity models discussed above, this contribution presents a step towards the enhancement of such theories.

${ }^{1}$ Lele and Anand implemented the theory by writing a nine-node quadratic user-element subroutine for the commercial finite element package ABAQUS/Standard. 
Moreover, an efficient discretization scheme suitable for parallelization has been suggested. The arising coupled boundary value problems were discretized with finite elements in space and the backward Euler in time. A dual mixed approach with displacements and gradient hardening variables as degrees of freedom is used. Such an approach is attractive since any crystal plasticity code (for the local problem) can be used by simply modifying the current yield stress due to the given gradient hardening variables in the nodes. The dual mixed finite element algorithm renders a coupled two-field problem which is solved monolithically. In particular, an algorithm suitable for parallelization was presented, where each grain is treated as a subproblem. A fast solution algorithm opens up for a variety of applications, since the resulting finite element problems tend to be rather large. Possible applications are concurrent multiscale modeling, or three dimensional models, for example.

In conclusion, the presented models and the numerical strategy are all suitable for handling, e.g., more advanced modeling of the hardening. Nevertheless, work remains to be done with respect to both, the modeling (e.g. an accurate prediction of the Hall-Petch effect) and the numerical implementation (regarding e.g. the nonstandard higher order dissipative term).

\section{Appendix: Thermodynamic considerations}

As mentioned above, there exists a thermodynamic setting for model II and III. First, the ideas of Ekh et al. [7] (model II) are presented and a short derivation for the definition of the hardening stresses $\kappa_{\alpha}$, i.e., Eq. (18), is given. Second, the considerations of Lele and Anand [17], see also Gurtin and Anand [14], are summarized.

In rational thermodynamics the second law imposes restrictions on constitutive equations. Whereas there exists a unified opinion about the balance laws, this is not the case for the second law of thermodynamics. Exploitations of the entropy inequality are based on distinctive mathematical procedures with the help of which conditions for a given class of constitutive material behaviors are derived in order to be compatible with the entropy inequality. Probably the best-known principle is that of Clausius and Duhem in the framework established by Coleman and Noll $[6]$.

The thermodynamic formulation of field and constitutive relations are ultimately formulated in the context of the total energy balance. Isothermal and quasi-static conditions are considered. In this case, the total energy balance reads

$$
\mathrm{D}_{t} \int_{\mathcal{B}_{0}} \Psi_{0} \mathrm{~d} V+\int_{\mathcal{B}_{0}} \mathcal{D}_{0} \mathrm{~d} V+\int_{\mathcal{B}_{0}} W_{\text {int }} \mathrm{d} V=0
$$

Here, $\Psi_{0}$ is the free energy per unit undeformed volume, $\mathcal{D}_{0}$ the dissipation power and $W_{\text {int }}$ stems from the rate of mechanical work supply. Although the balance equation is stated with respect to integrals over the entire material domain $\mathcal{B}_{0}$ respectively its entire boundary, it also has to hold for arbitrary bounded and connected parts of the body.

\section{Considerations of Ekh et al. [7, 23]}

The free energy $\Psi_{0}$ (per unit undeformed volume), in the material configuration $\mathcal{B}_{0}$, is proposed as a function of the deformation gradient $\boldsymbol{F}$ and a set of internal variables, which are here taken as $\boldsymbol{F}^{\mathrm{p}}$ and the set of slip-system shears $\left\{\gamma_{\alpha}\right\}_{\alpha=1}^{n_{\text {slip }}}$. In 
addition, the free energy $\Psi_{0}$ is assumed to depend on the set $\left\{\nabla_{\boldsymbol{X}} \gamma_{\alpha}\right\}_{\alpha=1}^{n_{\text {slip }}}$, where $\nabla_{\boldsymbol{X}}$ is the gradient in the reference configuration $\mathcal{B}_{0}$. Thus, gradient effects (under isothermal conditions) are included and the scalar variables $\gamma_{\alpha}$ represent isotropic hardening on the $n_{\text {slip }}$ slip-systems. From the principle of (spatial) objectivity, we may conclude that a possible parametrization of the free energy $\Psi_{0}$ is given by

$$
\Psi_{0}\left(\boldsymbol{F}, \boldsymbol{F}^{\mathrm{p}},\left\{\gamma_{\alpha}\right\},\left\{\nabla_{\boldsymbol{X}} \gamma_{\alpha}\right\}\right)=\bar{\Psi}_{0}\left(\overline{\boldsymbol{E}}^{\mathrm{e}},\left\{\gamma_{\alpha}\right\},\left\{\boldsymbol{\nabla}_{\boldsymbol{X}} \gamma_{\alpha}\right\}\right)
$$

Moreover, the the rate of mechanical work supply reads $W_{\text {int }} \stackrel{\text { def }}{=} \int_{\mathcal{B}_{0}} \boldsymbol{P}: \dot{\boldsymbol{F}} \mathrm{d} V$. For thermodynamical consistency, it is indispensable that the dissipation is strictly nonnegative, corresponding to the second law of thermodynamics. Thus, the fulfilment of the (reduced) dissipation inequality

$$
\int_{\mathcal{B}_{0}} \mathcal{D}_{0} \mathrm{~d} V=\int_{\mathcal{B}_{0}}\left[\overline{\boldsymbol{M}}^{\mathrm{e}}: \boldsymbol{L}^{\mathrm{p}}-\sum_{\alpha} \frac{\partial \bar{\Psi}_{0}}{\partial \gamma_{\alpha}} \dot{\gamma}_{\alpha}-\sum_{\alpha} \frac{\partial \bar{\Psi}_{0}}{\partial\left(\nabla_{\boldsymbol{X}} \gamma_{\alpha}\right)} \cdot \nabla_{\boldsymbol{X}} \dot{\gamma}_{\alpha}\right] \mathrm{d} V \geq 0
$$

is an absolutely essential modeling-feature. Upon integrating the last term by parts and using Gauss' theorem, we rewrite Eq. (38) as

$$
\int_{\mathcal{B}_{0}}\left[\overline{\boldsymbol{M}}^{\mathrm{e}}: \boldsymbol{L}^{\mathrm{p}}-\sum_{\alpha} \kappa_{\alpha} \dot{\gamma}_{\alpha}\right] \mathrm{d} V-\int_{\partial \mathcal{B}_{0}} \sum_{\alpha} \kappa_{\alpha}^{(b)} \dot{\gamma}_{\alpha} \mathrm{d} A \geq 0 .
$$

This leads to the definition of the auxiliary dissipative hardening stresses as stated in Eq. (18). It is noted that the hardening stresses $\kappa_{\alpha}$ may be composed of local as well as gradient contributions, whereas the gradient tractions $\kappa_{\alpha}^{(b)}$ on the boundary $\partial \mathcal{B}_{0, \text { grain }}$ represent the gradient effect only.

Note that a more restrictive formulation than Eq. (38) is obtained, if it is required that the local dissipation contributions are non-negative pointwise, i.e., if $\mathcal{D}_{0} \geq 0$ for all $\boldsymbol{X} \in \mathcal{B}_{0}$ and $\mathcal{D}_{0}^{(\mathrm{b})} \geq 0$ for all $\boldsymbol{X} \in \partial \mathcal{B}_{0}$.

\section{Considerations of Anand, Lele and Gurtin [14, 17]}

Anand, Lele and Gurtin $[14,17]$ apply a special kind of virtual work principle in conjunction with arguments of classical thermodynamics. Key idea is the assumption that each evolution of the body is associated with macroscopic and microscopic forces. The latter depends on a scalar microscopic force $f_{\alpha}$ whose working accompanies slip on slip-system $\alpha$ and a vector microscopic stress $\boldsymbol{\xi}_{\alpha}$ whose working accompanies slip gradients on $\alpha$. This leads to a different choice of an internal energy

$$
\begin{aligned}
W_{\text {int }} & \stackrel{\text { def }}{=} \int_{\mathcal{B}_{0}} \boldsymbol{S}^{\mathrm{e}}: \dot{\boldsymbol{E}}^{\mathrm{e}}+\sum_{\alpha}\left[f_{\alpha} \dot{\gamma}_{\alpha}+\boldsymbol{\xi}_{\alpha} \cdot \nabla_{\boldsymbol{X}} \dot{\gamma}_{\alpha}\right] \mathrm{d} V \\
& =\int_{\mathcal{B}_{0}} \boldsymbol{P}: \dot{\boldsymbol{F}}+\sum_{\alpha}\left[\operatorname{Div}\left(\boldsymbol{\xi}_{\alpha}\right) \dot{\gamma}_{\alpha}+\boldsymbol{\xi}_{\alpha} \cdot \nabla_{\boldsymbol{X}} \dot{\gamma}_{\alpha}\right] \mathrm{d} V
\end{aligned}
$$

with $\boldsymbol{S}^{\text {e }}$ expanding power over the Green-Lagrange strain $\dot{\boldsymbol{E}}^{\mathrm{e}}$. It is worth noting that, here, the work conjugacy of the scalar microscopic force $f_{\alpha}$ and the slip 
rate $\dot{\gamma}_{\alpha}$, respectively the microscopic stress $\boldsymbol{\xi}_{\alpha}$ and the slip rate gradient $\boldsymbol{\nabla}_{\boldsymbol{X}} \dot{\gamma}_{\alpha}$, is assumed first. Then the existence of a free energy function

$$
\Psi_{0}\left(\boldsymbol{F}, \boldsymbol{F}^{\mathrm{p}},\left\{\gamma_{\alpha}\right\},\left\{\nabla_{\boldsymbol{X}} \gamma_{\alpha}\right\}\right)=\bar{\Psi}_{0}\left(\overline{\boldsymbol{E}}^{\mathrm{e}},\left\{\nabla_{\boldsymbol{X}} \gamma_{\alpha}\right\}\right)
$$

is established. This is in contrast to the procedure described in Section 6, where first a free energy function depending on the slip-system shear $\gamma_{\alpha}$ and its gradient $\boldsymbol{\nabla}_{\boldsymbol{X}} \gamma_{\alpha}$ is assigned which then, as a consequence, leads to the definition of microscopic hardening stresses $\kappa_{\alpha}$, i.e., Eq. (18). Moreover, the free energy function of Anand, Lele and Gurtin $[14,17]$ does not depend on the slip-system shear $\gamma_{\alpha}$, only on its gradient.

Therefore, the counterpart of dissipation inequality (38) reads

$$
\int_{\mathcal{B}_{0}}\left[\overline{\boldsymbol{M}}^{\mathrm{e}}: \boldsymbol{L}^{\mathrm{p}}+\sum_{\alpha}\left[\operatorname{Div}\left(\boldsymbol{\xi}_{\alpha}\right) \dot{\gamma}_{\alpha}+\boldsymbol{\xi}_{\alpha} \cdot \nabla_{\boldsymbol{X}} \dot{\gamma}_{\alpha}\right]\right] \mathrm{d} V \geq 0 .
$$

The strategy of Anand, Lele and Gurtin leads to the microforce balance, i.e., Eq. (24), where an additive decomposition of the microstress $\boldsymbol{\xi}_{\alpha}$ into an energetic and a dissipative component is admitted

$$
\boldsymbol{\xi}_{\alpha}=\boldsymbol{\xi}_{\alpha}^{\mathrm{en}}+\boldsymbol{\xi}_{\alpha}^{\mathrm{diss}}
$$

The energetic microscopic stress for slip-system $\alpha$, i.e., $\boldsymbol{\xi}_{\alpha}^{\mathrm{en}}$, is given by

$$
\boldsymbol{\xi}_{\alpha}^{\text {en }} \stackrel{\partial \overline{d e f}_{0}}{=} \frac{\partial \bar{\Psi}_{0}}{\partial\left(\nabla_{\boldsymbol{X}} \gamma_{\alpha}\right)}
$$

Then, the dissipation takes the form

$$
\sum_{\alpha}\left[f_{\alpha} \dot{\gamma}_{\alpha}+\boldsymbol{\xi}_{\alpha}^{\text {diss }} \cdot \nabla_{\boldsymbol{X}} \dot{\gamma}_{\alpha}\right] \mathrm{d} V \geq 0
$$

Inequality (45) imposes restrictions on the constitutive equations for $f_{\alpha}$ and $\boldsymbol{\xi}_{\alpha}^{\text {diss }}$ for which a constitutive dependence on the slip rate $\dot{\gamma}_{\alpha}$ and the slip rate gradient $\nabla_{\boldsymbol{X}} \dot{\gamma}_{\alpha}$ is suggested, cf. $[14,17]$.

In addition, since the microscopic stress $\boldsymbol{\xi}_{\alpha}^{\text {diss }}$ characterizes dissipative microscopic forces associated with the evolution of dislocations on the $\alpha$ th slip plane, and because the motion of such dislocations is tangent to this plane, it sometimes is required that dissipative microscopic stress $\boldsymbol{\xi}_{\alpha}^{\text {diss }}$ is tangential, cf. e.g. [14].

\section{Acknowledgement}

This work was supported by the Swedish Research Council, which is gratefully acknowledged. This research was carried out while Swantje Bargmann was at Chalmers University, Sweden, as a post-doctoral fellow. The authors are very grateful to the anonymous referees for their helpful comments and suggestions leading to the improvement of this contribution. 


\section{References}

[1] E.C. Aifantis, On the microstructural origin of certain inelastic models. J. Eng. Mater. Technol. 106 (1984) pp. 326-330.

[2] M.F. Ashby, The deformation of plastically non-homogeneous materials. Philos. Mag. 21 (1970) pp. 399-424.

[3] J.L. Bassani, A. Needleman, E. Van der Giessen, Plastic flow in a composite: a comparison of nonlocal continuum and discrete dislocation predictions. International Journal of Solids and Structures 38 (2001), pp. 833-853

[4] C.J. Bayley, W.A.M. Brekelmans, M.G.D. Geers, A three-dimensional dislocation field crystal plasticity approach applied to miniaturized structures. Philos. Mag. 87 (2007) pp. 1361-1378

[5] E. Bittencourt, A. Needleman, M.E. Gurtin, E. Van der Giessen, A comparison of nonlocal continuum and discrete dislocation plasticity predictions. Journal of the Mechanics and Physics of Solids 51 (2003) pp. 281-310

[6] B.D. Coleman, W. Noll The thermodynamics of elastic materials with heat conduction and viscosity. Archive for Rational Mechanics and Analysis 13 (1963) pp. 167-178

[7] M. Ekh, M. Grymer, K. Runesson, T. Svedberg, Gradient crystal plasticity as part of the computational modeling of polycrystals. International Journal for Numerical Methods in Engineering 72 (2007) pp. 197-220

[8] U. Essmann, H. Mughrabi, Annihilation of dislocations during tensile and cyclic deformation and limits of dislocation densities. Philos. Mag. A 40 (1979) pp. 731-756

[9] L.P. Evers, W.A.M. Brekelmanns, M.G.D. Geers, Non-local crystal plasticity model with intrinsic SSD and GND effects. Journal of the Mechanics and Physics of Solids 52 (2004) pp. 2379-2401

[10] S. Forest, R. Sedláček, Plastic slip distribution in two-phase laminate microstructures: dislocationbased versus generalized-continuum approaches. Philosophical Magazine 83 (2003) pp. 245-276

[11] S. Forest, Some links between Cosserat, strain gradient crystal plasticity and the statistical theory of dislocations. Philosophical Magazine 88 (2008) pp. 3549-3563

[12] P. Fredriksson, P. Gudmundson, Size-dependent yield strength of thin films. International Journal of Plasticity 21 (2005) pp. 1834-1854

[13] M.E. Gurtin, A gradient theory of small-deformation isotropic plasticity that accounts for the Burgers vector and for dissipation due to plastic spin. Journal of the Mechanics and Physics of Solids 52 (2004) pp. 2545-2568

[14] M.E. Gurtin, L. Anand, A theory of strain-gradient plasticity for isotropic, plastically irrotational materials. Part I: Small deformations. Journal of the Mechanics and Physics of Solids 53 (2005) pp. 1624-1649

[15] E.O. Hall, The deformation and ageing of mild steel: III discussion of results. Proc. Phys. Soc. Lond. B 64 (1951) pp. 747-753

[16] A. Hunter, M. Koslowski, Direct calculations of material parameters for gradient plasticity. Journal of the Mechanics and Physics of Solids 56 (2008) pp. 3181-3190

[17] S.P. Lele, L. Anand, A small-deformation strain-gradient theory for isotropic viscoplastic materials Philosophical Magazine (2008), DOI: 10.1080/14786430802087031

[18] V. Levkovitch, B. Svendsen, On the large-deformation- and continuum-based formulation of models for extended crystal plasticity International Journal of Solids and Structures 43 (2006) pp. 7246-7267

[19] T. Liebe, P. Steinmann, Theory and numerics of a thermodynamically consistent framework for geometrically linear gradient plasticity International Journal for Numerical Methods in Engineering 51 (2001) pp. 1437-1467

[20] N.J. Petch, The cleavage strength of polycrystals. I J. Iron and Steel Institute 174 (1953) pp. 25-28

[21] J.R. Rice, Inelastic constitutive relations for solids: an internal variable theory and its application to metal plasticity. J.Mech.Phys.Solids 19 (1971) pp. 433-455

[22] T. Svedberg, K. Runesson, A thermodynamically consistent theory of gradient-regularized plasticity coupled to damage. International Journal of Plasticity 13 (1997) pp. 669-696

[23] T. Svedberg, K. Runesson, An algorithm for gradient-regularized plasticity coupled to damage based on a dual mixed FE-formulation Computer Methods in Applied Mechanics and Engineering 161 (1998) pp. 49-65

[24] B. Svendsen, Continuum thermodynamic extensions of crystal plasticity to include the effects of geometrically-necessary dislocations on the material behaviour Rend. Sem. Mat. Univ. Pol. Torino, 58 (2000) pp. 209-235

[25] B. Svendsen, Continuum thermodynamic models for crystal plasticity including the effects of geometrically-necessary dislocations J. Mech. Phys. Solids, 50 (2002) pp. 1297-1329

[26] B. Svendsen, On thermodynamic and variational-based formulations of models for inelastic continua with internal lengthscales Computer Methods in Applied Mechanics and Engineering 193 (2004) pp. 5429-5452

[27] S.M. Vrech, G. Etse, FE approach for thermodynamically consistent gradient-dependent plasticity Latin American Applied Research 37 (2007) pp. 127-132 

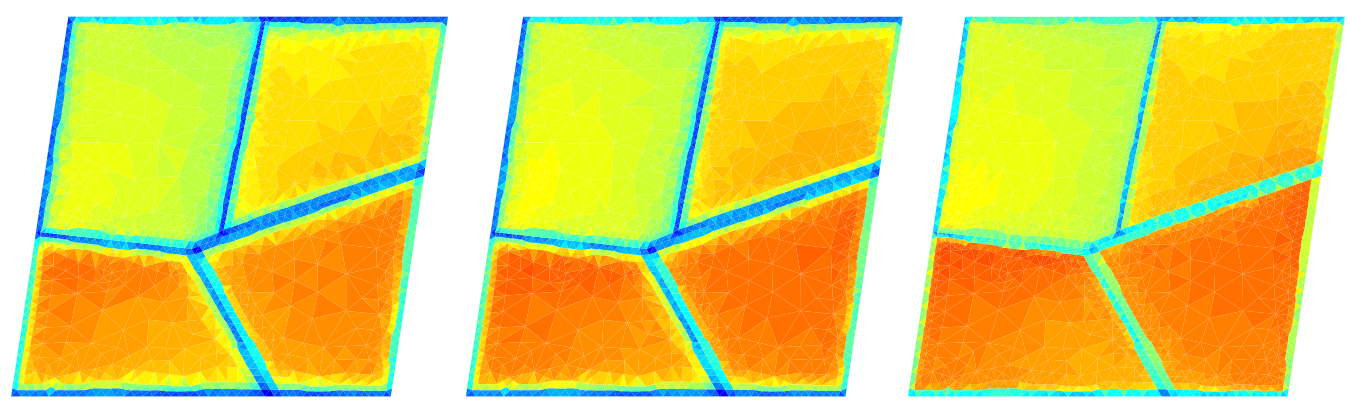

Figure 1. Left: Discretization and grain geometry used during simulations. The square grain-structure consists of 4 grains and the thick black lines represent the grain boundaries. The grains are discretized with 726 (upper left grain), 620 (upper right), 630 (lower left), and 614 (lower right) elements, respectively. The mesh is refined near the grain boundaries. The side length $L$ of the grain-structure is varied during simulations in order to capture the size effects.

Right: Schematic sketch of slip plane directions $\boldsymbol{s}_{\alpha}$. Double slip is assumed and slip plane directions are randomly distributed.

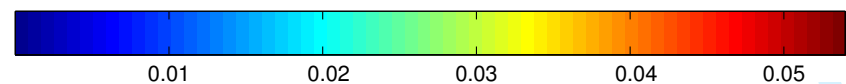

Figure 2. Model I, Evers et al. [9]. Effective hardening strain $\gamma_{\mathrm{eff}} \stackrel{\text { def }}{=} \sqrt{\gamma_{1}^{2}+\gamma_{2}^{2}+\gamma_{3}^{2}+\gamma_{4}^{2}}$. From left to right the solutions for grain-structure side lengths $L=4 \mu \mathrm{m}, L=8 \mu \mathrm{m}$, and $L=16 \mu \mathrm{m}$ are depicted. The evolution of the slip-system shear is given in Eq. (12). Computations are carried out in monotonic loading with a maximum macroscopic shear deformation $\bar{\gamma}_{\max }=0.15$. The displacement field inside the grainstructure is unconstrained. The slip resistance $s_{\alpha}$ is computed according to Eq. (16), the rate sensitivity parameter reads $m=1$. 


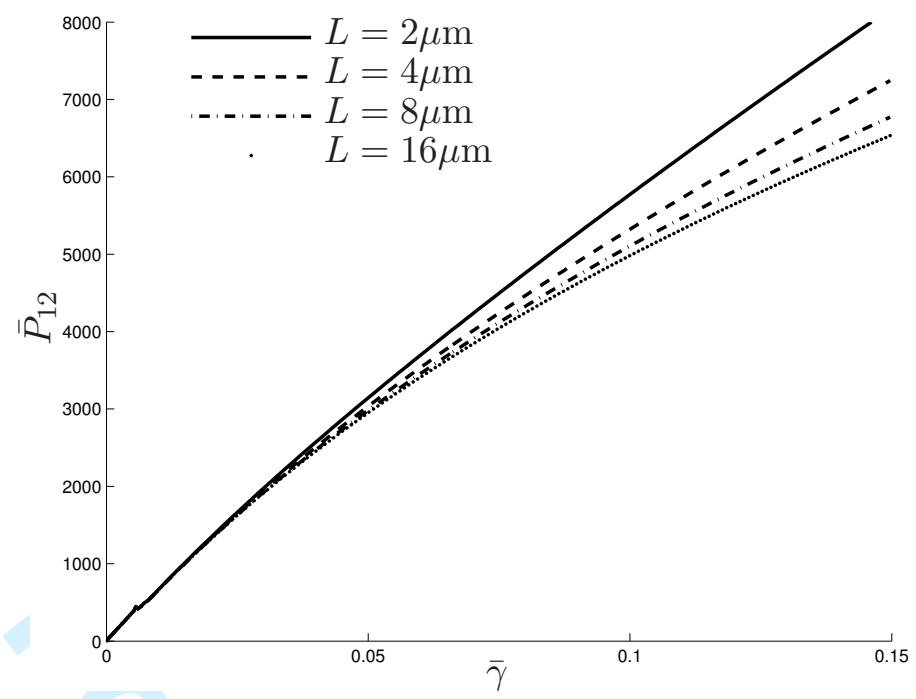

Figure 3. Model I, Evers et al. [9]. Macroscopic stress-strain response $\left(\bar{P}_{12}\right.$ vs. $\left.\bar{\gamma}\right)$ showing the size dependence on the amount of hardening. Under the assumption of increasing slip resistance $s_{\alpha}$ according to Eq. (16) and $m=1$.
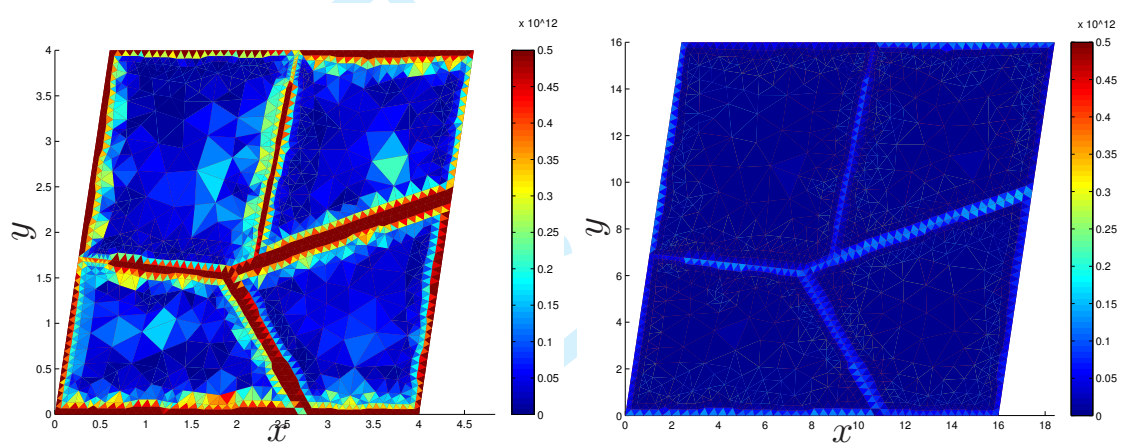

Figure 4. Model I, Evers et al. [9]. Slip-system 1 GND densities are plotted for two samples lengths after applying $15 \%$ shear. One the left the distribution of the GND density $\rho_{\text {GND }}$ for a grain structure with $L=4 \mu \mathrm{m}$ and on the right with $L=16 \mu \mathrm{m}$ can be seen. A size-dependence is found for the density distribution. Under the assumption of increasing slip resistance $s_{\alpha}$ according to Eq. (16) and $m=1$. The sign of the $\rho_{\mathrm{GND}}$ is ignored in this plot. The results are in accordance to [9].
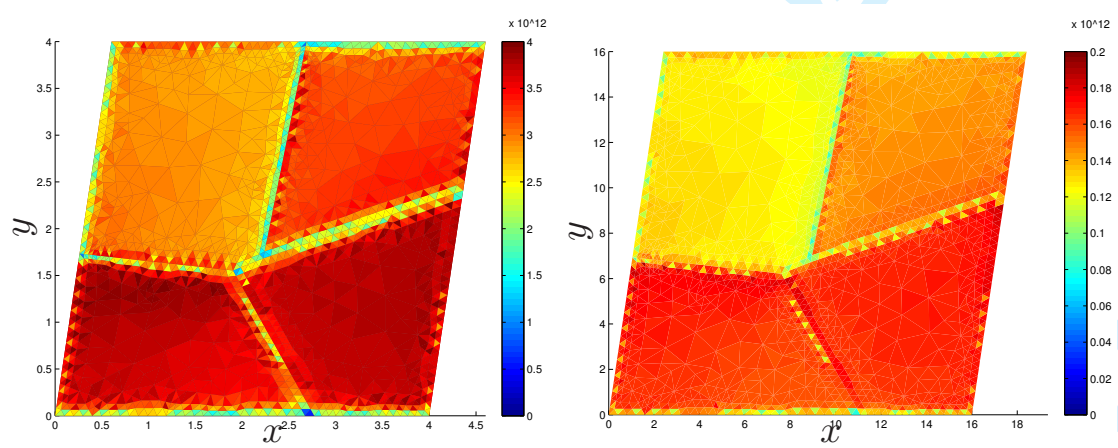

Figure 5. Model I, Evers et al. [9]. Slip-system 1 SSD densities are illustrated for two samples lengths after applying $15 \%$ shear. One the left the distribution of the SSD density $\rho_{\mathrm{SSD}}$ for a grain structure with $L=4 \mu \mathrm{m}$ and on the right with $L=16 \mu \mathrm{m}$ can be seen. $\rho_{\mathrm{SSD}}$ decreases towards the boundary. Under the assumption of increasing slip resistance $s_{\alpha}$ according to Eq. (16) and $m=1$. The results are in accordance to $[9]$. 

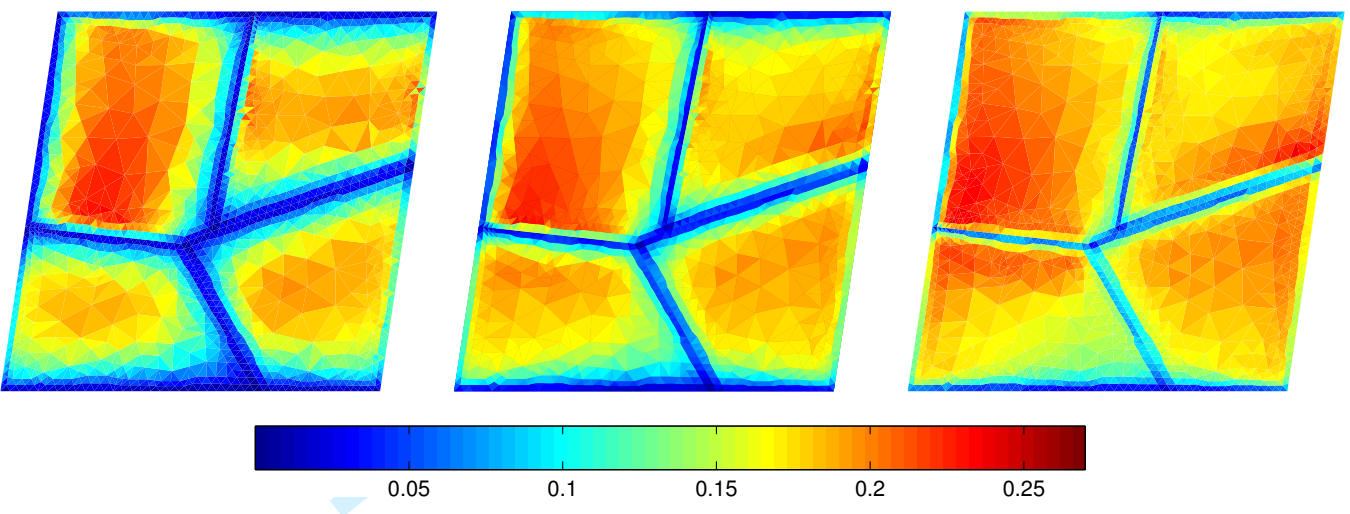

Figure 6. Model I, Evers et al. [9]. Effective hardening strain $\gamma_{\text {eff }} \stackrel{\text { def }}{=} \sqrt{\gamma_{1}^{2}+\gamma_{2}^{2}+\gamma_{3}^{2}+\gamma_{4}^{2}}$. From left to right the accumulated plastic strain field for grain-structure side lengths $L=4 \mu \mathrm{m}, L=8 \mu \mathrm{m}$, and $L=16 \mu \mathrm{m}$ are depicted. The evolution of the slip-system shear is given in Eq. (12). Computations are carried out in monotonic loading with a maximum macroscopic shear deformation $\bar{\gamma}_{\max }=0.15$. The displacement field inside the grain-structure is unconstrained. The slip resistance $s_{\alpha}$ is assumed to be constant with $s_{\alpha}=C_{0}=1[\mathrm{MPa}]$ and $m=1$.

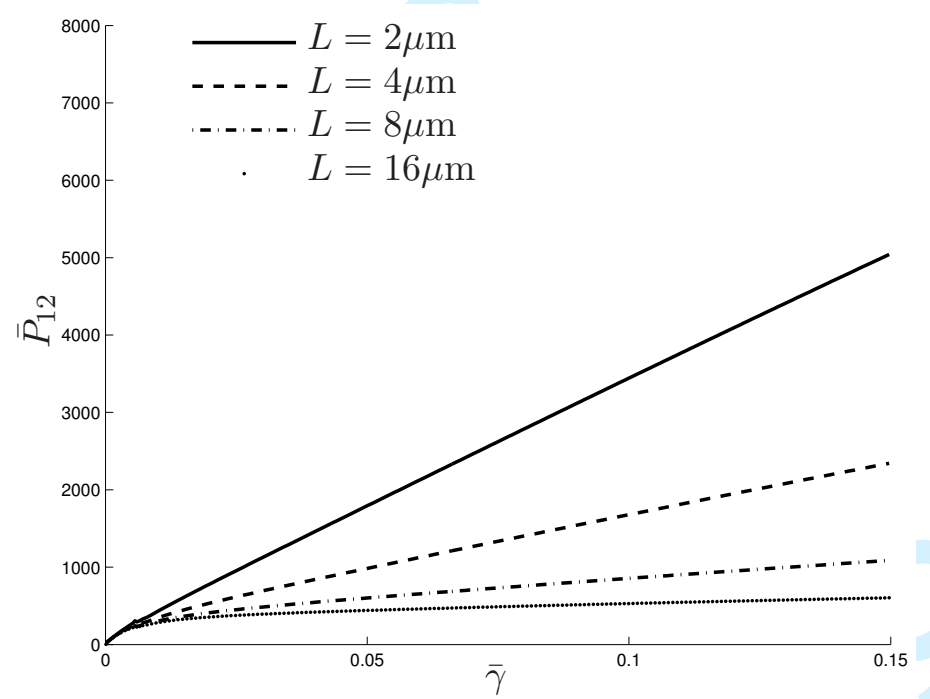

Figure 7. Model I, Evers et al. [9]. Macroscopic stress-strain response ( $\bar{P}_{12}$ vs. $\left.\bar{\gamma}\right)$ showing the size dependence on the amount of hardening. $m=1$. Constant slip resistance $s_{\alpha}=C_{0}=1$ [MPa] presumed, i.e., no local contribution to the hardening exists. The grain-structure still shows a size-dependent response. 

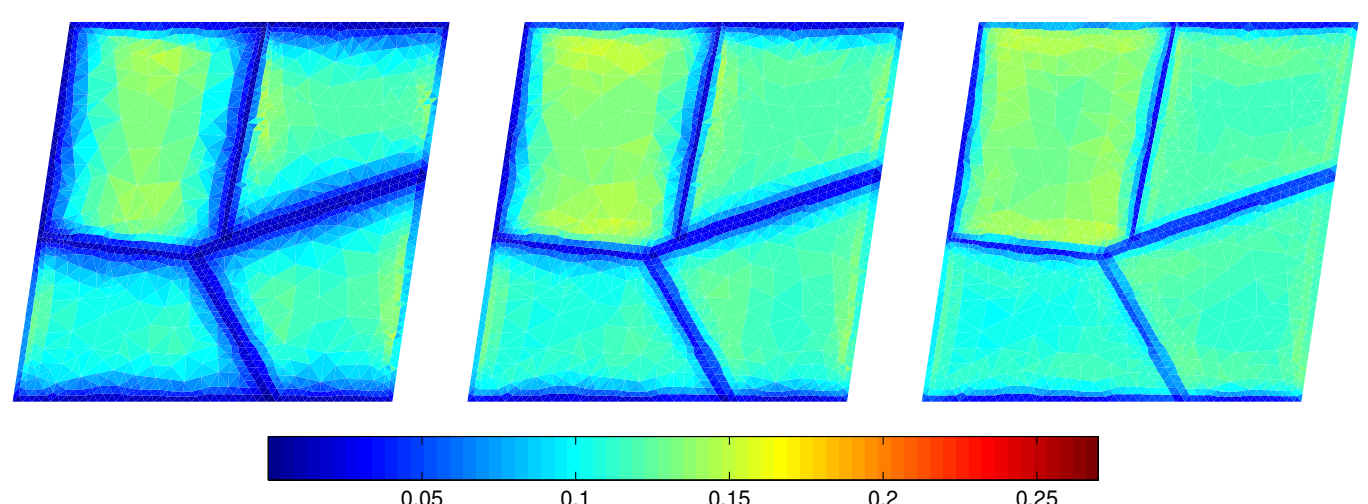
pendence on the amount of hardening. Local hardening is admitted. The rate sensitivity parameter $m$ is raised to $m=5$ (left) and $m=20$ (right). The higher the rate sensitivy parameter $m$, the softer responds the material.

Figure 9. Model II, Ekh et al. [7]. Effective hardening strain $\gamma_{\text {eff }}=\sqrt{\gamma_{1}^{2}+\gamma_{2}^{2}+\gamma_{3}^{2}+\gamma_{4}^{2}}$. From left to right the accumulated plastic strain field for grain-structure side lengths $L=4 \mu \mathrm{m}, L=8 \mu \mathrm{m}$, and $L=16 \mu \mathrm{m}$ are depicted. The evolution of the slip-system shear $\gamma_{\alpha}$ is given in Eq. (19) - with an initial yield stress $Y_{\alpha}=10^{3}[\mathrm{MPa}]$ and a local hardening module $H_{\alpha}^{l}=10^{4}[\mathrm{MPa}]$ for all slip-systems $\alpha=1, \ldots, 4$. The rate sensitivity parameter is set at $m=1$. The displacement field inside the grain-structure is unconstrained. Since the (absolute) size of the boundary layers with large gradient effects is approximately the same regardless of the actual size of the grains, the boundary layers appear thinner as the grain size increases. 


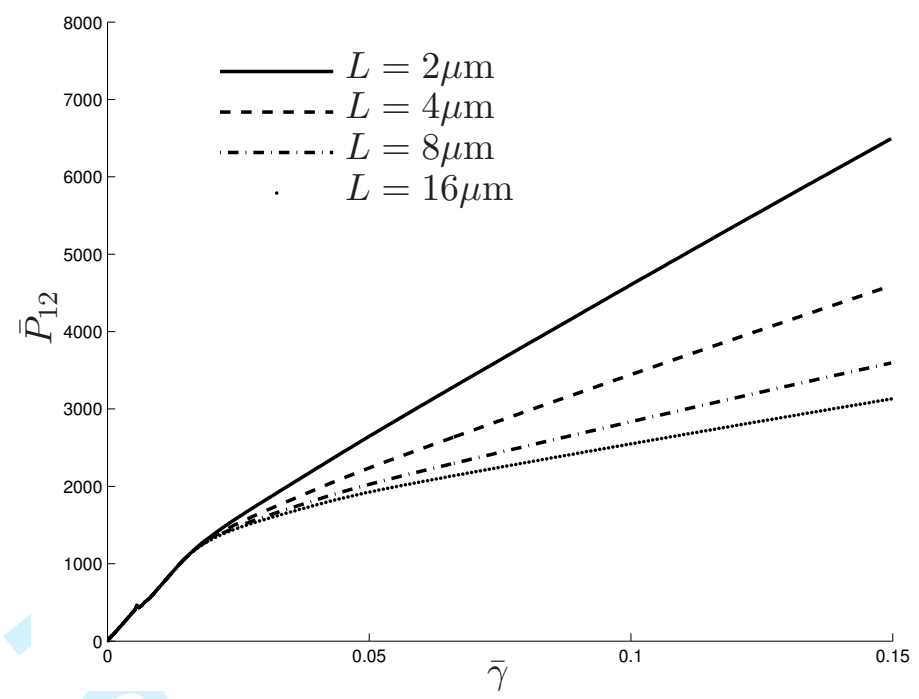

Figure 10. Model II, Ekh et al. [7]. Initial yield stress $Y_{\alpha}=10^{3}$ [MPa], local hardening module $H_{\alpha}^{l}=$ $10^{4}[\mathrm{MPa}]$ for all slip-systems $\alpha=1, \ldots, 4, m=1$.. Macroscopic stress-strain response $\left(\bar{P}_{12}\right.$ vs. $\left.\bar{\gamma}\right)$ showing the size dependence on the amount of hardening.
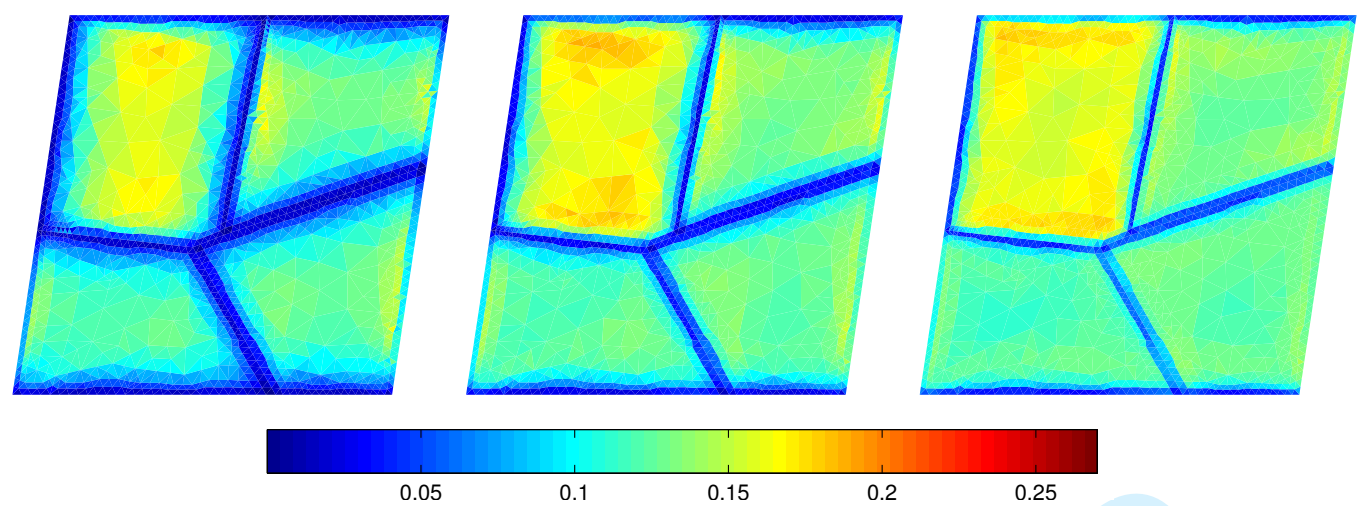

Figure 11. Model II, Ekh et al. [7]. Effective hardening strain $\gamma_{\mathrm{eff}}=\sqrt{\gamma_{1}^{2}+\gamma_{2}^{2}+\gamma_{3}^{2}+\gamma_{4}^{2}}$. The accumulated plastic strain field for grain-structure side lengths $L=4 \mu \mathrm{m}, L=8 \mu \mathrm{m}$, and $L=16 \mu \mathrm{m}$ (from left to right) are depicted. The evolution of the slip-system shear $\gamma_{\alpha}$ is given in Eq. (19) - with an initial yield stress $Y_{\alpha}=0[\mathrm{MPa}]$ and a local hardening module $H_{\alpha}^{l}=10^{4}[\mathrm{MPa}]$ for all slip-systems $\alpha=1, \ldots, 4$. Rate sensitivity parameter $m=1$. The displacement field inside the grain-structure is unconstrained. It is observed that the non-existence of the initial yield stress $Y_{\alpha}$ leads to plastic yielding from the beginning of the deformation, see Figure 9 for comparison. 


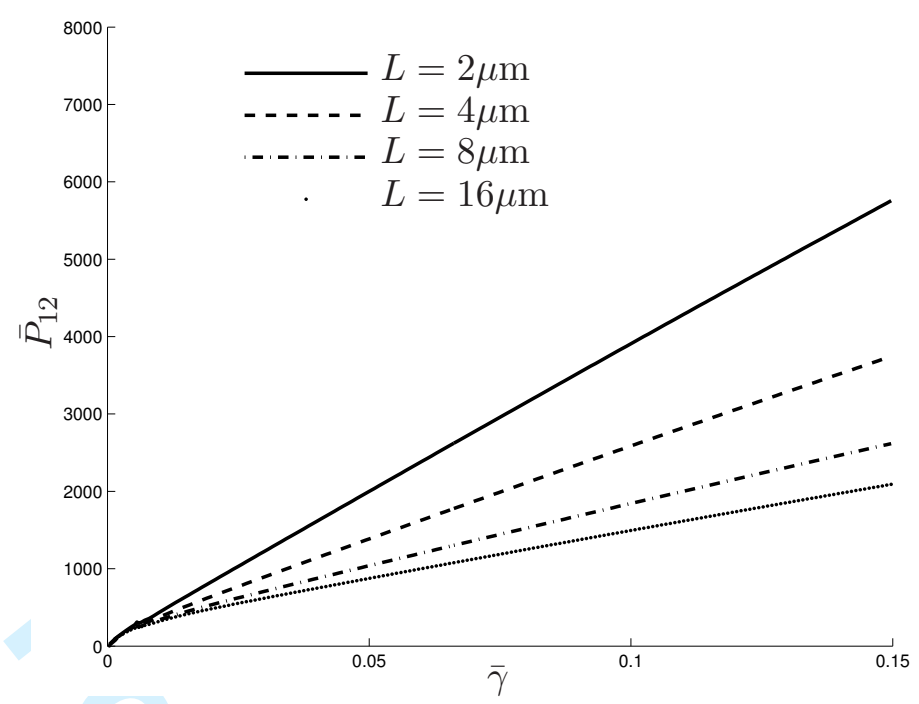

Figure 12. Model II, Ekh et al. [7]. Initial yield stress $Y_{\alpha}=0[\mathrm{MPa}]$, local hardening module $H_{\alpha}^{l}=$ $10^{4}[\mathrm{MPa}]$ for all slip-systems $\alpha=1, \ldots, 4, m=1$. Macroscopic stress-strain response $\left(\bar{P}_{12}\right.$ vs. $\left.\bar{\gamma}\right)$ showing the size dependence on the amount of hardening and the influence of the initial yield stress $Y_{\alpha}$, see Figure 10 for comparison.

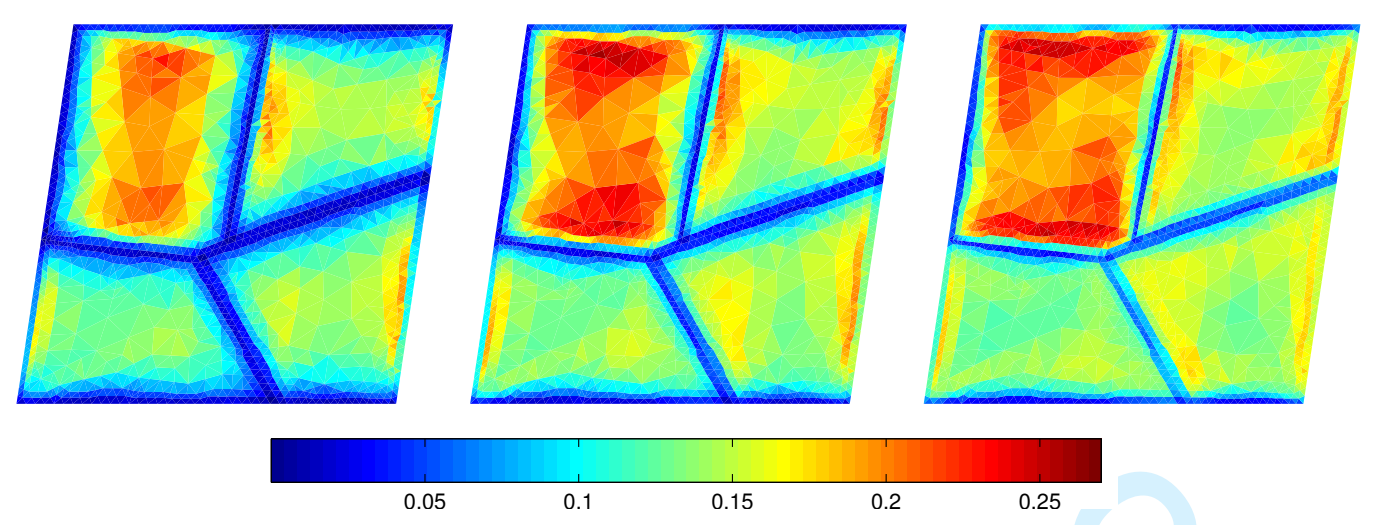

Figure 13. Model II, Ekh et al. [7]. Effective hardening strain $\gamma_{\text {eff }}=\sqrt{\gamma_{1}^{2}+\gamma_{2}^{2}+\gamma_{3}^{2}+\gamma_{4}^{2}}$. From left to right the solutions for RVE side lengths $L=4 \mu \mathrm{m}, L=8 \mu \mathrm{m}$, and $L=16 \mu \mathrm{m}$ are depicted. The evolution of the slip-system shear $\gamma_{\alpha}$ is given in Eq. (19) - with an initial yield stress $Y_{\alpha}=0$ [MPa] and a local hardening module $H_{\alpha}^{l}=0$ [MPa] for all slip-systems $\alpha=1, \ldots, 4 . m=1$. The displacement field inside the grain-structure is unconstrained. Neglecting the local hardening module $H_{\alpha}^{l}$ leads to significantly more slip inside the grain-structure, see Figures 9 and 11 for comparison. 


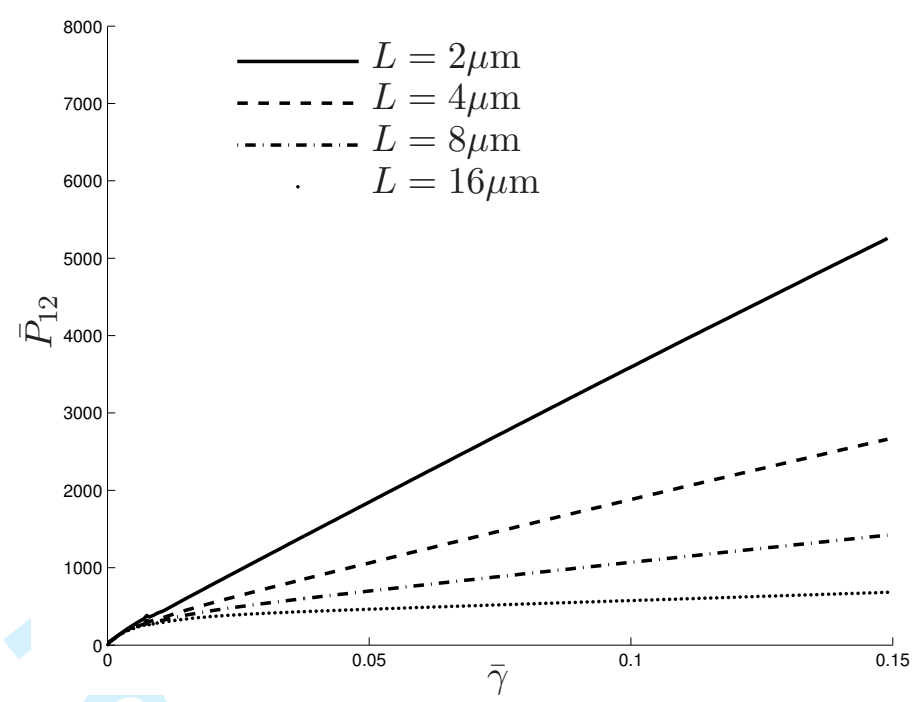

Figure 14. Model II, Ekh et al. [7]. Initial yield stress $Y_{\alpha}=0$ [MPa], local hardening moduli $H_{\alpha}^{l}=0$ [MPa] for all slip-systems $\alpha=1, \ldots, 4$. Macroscopic stress-strain response $\left(\bar{P}_{12}\right.$ vs. $\left.\bar{\gamma}\right)$ showing the size dependence on the amount of hardening in case of vanishing local hardening moduli $H_{\alpha}^{l}$. Again, it can be stated that smaller the grain-structure size the stiffer is the material's response. However, it can clearly be seen that $H_{\alpha}^{l}=0[\mathrm{MPa}]$ leads to a significant decrease in the stiffness for all lengths $L . m=1$.
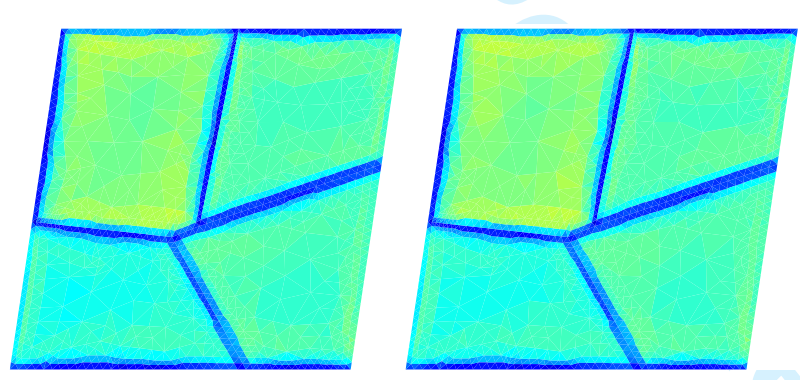

Figure 15. Model III. Effective hardening strain $\gamma_{\mathrm{eff}}=$ $\sqrt{\gamma_{1}^{2}+\gamma_{2}^{2}+\gamma_{3}^{2}+\gamma_{4}^{2}}$. Left the accumulated plastic strain field according to model III with $l_{d}=0 \mu \mathrm{m}$ for a grain-structure side length $16 \mu \mathrm{m}$ is depicted. On the right, the accumulated plastic strain field according with $l_{d}=0.06 \mu \mathrm{m}$ is shown. Computations are carried out in monotonic loading with a maximum macroscopic shear deformation $\bar{\gamma}_{\max }=0.15$. The evolution of the slip-system shear $\gamma_{\alpha}$ is given in Eq. (20) - with an initial yield stress $Y_{\alpha}=10^{3}[\mathrm{MPa}]$ and a local hardening module $H_{\alpha}^{l}=10^{4}[\mathrm{MPa}]$ for all slip-systems $\alpha=1, \ldots, 4$. The influence of the divergence term is very small, compare Figures 16 and 17 . The displacement field inside the grain-structure is unconstrained and $m=1$.

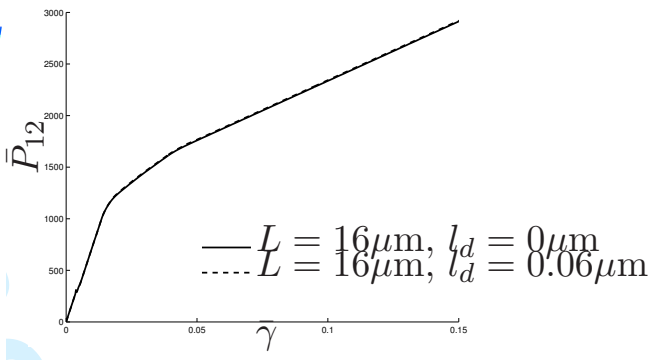

Figure 16. Model III. Initial yield stress $Y_{\alpha}=10^{3}[\mathrm{MPa}]$, local hardening module $H_{\alpha}^{l}=10^{4}[\mathrm{MPa}]$ for all slip-systems $\alpha=1, \ldots, 4, m=1$. Macroscopic stressstrain response $\left(\bar{P}_{12}\right.$ vs. $\left.\bar{\gamma}\right)$. The influence of the divergence term can hardly been seen in this example. 


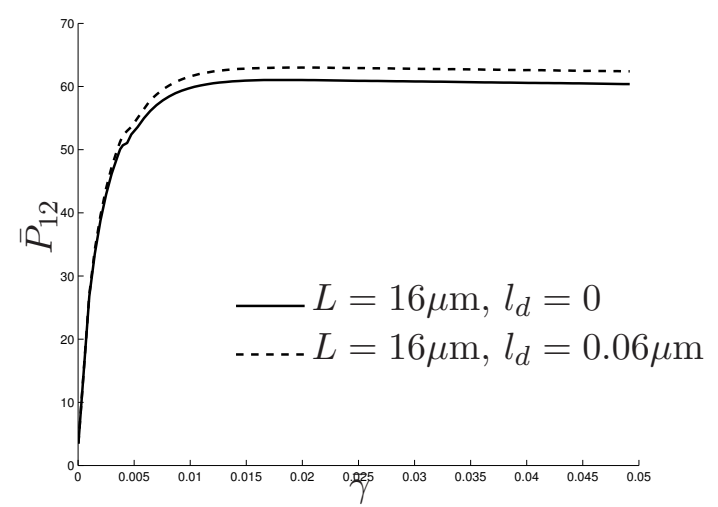

Figure 17. Model III. Initial yield stress $Y_{\alpha}=0[\mathrm{MPa}]$, local hardening module $H_{\alpha}^{l}=0[\mathrm{MPa}]$ for all slip-systems $\alpha=1, \ldots, 4$. Macroscopic stress-strain response $\left(\bar{P}_{12}\right.$ vs. $\left.\bar{\gamma}\right)$ showing the influence of the divergence term. Since the influence is governed by the dissipative length scale $l_{d}$, this material parameter has been varied. $m=1$. Note that strain is only plotted up to $0.05 \%$.

\begin{tabular}{|c|c|c|c|c|c|c|}
\hline Parameter & Symbol & Value & & & & \\
\hline Young's modulus & $E$ & $2 \cdot 10^{5}$ & {$[\mathrm{MPa}]$} & & & \\
\hline Poisson's ratio & $\nu$ & 0.3 & & & & \\
\hline local hardening modulus & $H_{\alpha}^{l}$ & $10^{4}$ & {$[\mathrm{MPa}]$} & resp & 0 & {$[\mathrm{MPa}]$} \\
\hline gradient hardening modulus & $H_{\alpha}^{g}$ & $4 \cdot 10^{7}$ & {$[\mathrm{MPa}]$} & & & \\
\hline internal length scale & $l_{\alpha}$ & $10^{-2}$ & {$[\mathrm{~mm}]$} & & & \\
\hline initial yield stress & $Y_{\alpha}$ & $10^{3}$ & {$[\mathrm{MPa}]$} & resp & 0 & {$[\mathrm{MPa}]$} \\
\hline relaxation time & $t_{*}$ & $10^{4}$ & & & & \\
\hline material constant & $C_{0}$ & 1 & {$[\mathrm{MPa}]$} & & & \\
\hline material constant & $c$ & 0.3 & & & & \\
\hline reference plastic strain rate & $\dot{\gamma}_{0}$ & 0.0001 & {$\left[\mathrm{~s}^{-1}\right]$} & & & \\
\hline rate sensitivity parameter & $m$ & 1 & & resp & 0.02 & \\
\hline strain rate sensitivity parameter & $q$ & 1 & & & & \\
\hline magnitude of Burger's vector & $b$ & 0.256 & {$[\mathrm{~nm}]$} & & & \\
\hline immobilization coefficient & $h_{0}$ & 1 & & & & \\
\hline interaction coefficient & $a_{0}$ & 0.06 & & & & \\
\hline radius GND evaluation region & $R$ & $10^{-1}$ & {$[\mathrm{~mm}]$} & & & \\
\hline critical annihilation length & $y_{c}$ & 1.6 & {$[\mathrm{~nm}]$} & & & \\
\hline initial SSD density & $\rho^{\mathrm{SSD}_{0}}$ & $7 \cdot 10^{12}$ & {$\left[\mathrm{~m}^{-2}\right]$} & & & \\
\hline initial GND density & $\rho^{\mathrm{GND}_{0}}$ & & {$\left[\mathrm{~m}^{-2}\right]$} & & & \\
\hline material constant & $K$ & 25 & & & & \\
\hline initial slip resistance & ${ }^{0} s_{\alpha}=s_{\alpha}(t=0)$ & 3.7 & {$[\mathrm{MPa}]$} & & & \\
\hline $\begin{array}{l}\text { dissipative length scale } \\
\text { reference rate }\end{array}$ & $\begin{array}{l}l_{d} \\
0_{d}\end{array}$ & $\begin{array}{l}0.06 \\
0.0001\end{array}$ & $\begin{array}{l}{[\mu \mathrm{m}]} \\
{\left[\mathrm{s}^{-1}\right]}\end{array}$ & resp & 0 & {$[\mu \mathrm{m}]$} \\
\hline & & & & & & \\
\hline
\end{tabular}

Table 1. Material parameters. The data is taken from [7], [8], [9] and [17]. Some of the values are adjusted in such a way that comparison between the models is possible, see also the remark on this aspect. During simulations some of the parameters are varied in order to study their influence in the material behavior. 